\title{
ANALYZING POLICY CAPTURING DATA USING STRUCTURAL EQUATION MODELING FOR WITHIN-SUBJECT EXPERIMENTS (SEMWISE)
}

Bert Weijters

Hans Baumgartner

Weijters, B., \& Baumgartner, H. (2018). Analyzing Policy Capturing Data Using Structural Equation Modeling for Within-Subject Experiments (SEMWISE). Organizational Research Methods, in press, 1094428118756742.

Bert Weijters is Associate Professor of Market Research, Department of Personnel Management, Work and Organizational Psychology, Ghent University, B-9000 Ghent, Belgium, T.: +32926462 96, Fax: +32926464 94, E-mail: bert.weijters@ugent.be.

Hans Baumgartner is the Smeal Professor of Marketing in the Smeal College of Business at The Pennsylvania State University, Department of Marketing, 482 Business Building, University Park, PA 16802, T. 814863 3559, E-mail: hansbaumgartner@psu.edu. 


\title{
Analyzing Policy Capturing Data Using Structural Equation Modeling for Within-Subject Experiments (SEMWISE)
}

\begin{abstract}
We present the SEMWISE (Structural Equation Modeling for Within-Subject Experiments) approach for analyzing policy capturing data. Policy capturing entails estimating the weights (or utilities) of experimentally manipulated attributes in predicting a response variable of interest (e.g., the effect of experimentally manipulated market-technology combination characteristics on perceived entrepreneurial opportunity). In the SEMWISE approach, a factor model is specified in which latent weight factors capture individually varying effects of experimentally manipulated attributes on the response variable. We describe the core SEMWISE model and propose several extensions (how to incorporate non-binary attributes and interactions; how to model multiple indicators of the response variable; how to relate the latent weight factors to antecedents and/or consequences; and how to simultaneously investigate several populations of respondents). The primary advantage of the SEMWISE approach is that it facilitates the integration of individually varying policy capturing weights into a broader nomological network while accounting for measurement error. We illustrate the approach with two empirical examples, compare and contrast the SEMWISE approach with multi-level modeling (MLM), discuss how researchers can choose between SEMWISE and MLM, and provide implementation guidelines.
\end{abstract}

Key words: Structural Equation Modeling; Within-Subject Experiments; Multi-level data; Policy Capturing; Conjoint Analysis. 
Organizational researchers often want to determine which pieces of information most strongly influence preferences or decisions (Aiman-Smith, Scullen, \& Barr, 2002). Such questions can be addressed through policy capturing designs. Policy capturing designs are in essence within-subject experiments that test how different combinations of attribute levels of stimuli influence individuals' responses. Policy capturing (also known as conjoint analysis) has been used in hundreds of judgment and decision-making studies in a variety of disciplines, including entrepreneurship, strategy, marketing, and organizational behavior (Shepherd, Patzelt, \& Baron, 2013). For instance, Grégoire and Shepherd (2012) use policy capturing to examine the influence of superficial and structural similarity between a technology and a potential target market on perceived entrepreneurial opportunity (i.e., the perceived fit of the technology with the market and its perceived feasibility). Aiman-Smith, Bauer, and Cable (2001) use policy capturing to examine the relative importance of four attributes (pay, promotion opportunities, lay-off policy and a firm's ecological rating) on potential job recruits' attraction to an organization and job pursuit intentions. Table 1 presents an overview of these and some additional examples.

The analysis of policy capturing studies can take diverse forms. While these designs were traditionally analyzed using ANOVA or regression analysis (Aiman-Smith et al., 2002), more recently multi-level modeling (MLM) has become the recommended analysis technique (Aguinis \& Bradley, 2014). As we argue in this paper, even though Structural Equation Modeling (SEM) is currently not being employed for analyzing policy capturing data, it could be used for this purpose. The aim of the present paper is to address this gap by introducing Structural Equation Modeling for Within-Subject Experiments, abbreviated SEMWISE.

As discussed in more detail later, the SEMWISE approach offers certain advantages over alternative methodologies in specific circumstances. In general, it provides a flexible yet rigorous analytical framework in which individually varying responses to different attributes 
of experimental stimuli are modeled as latent variables. These latent variables can be easily integrated into a broader nomological network of antecedents and consequences. Further, as in any SEM application, detailed model fit information is available, so the specified model can be thoroughly assessed and tested against alternative specifications, and parameter restrictions can be easily evaluated based on indices of local misfit (e.g., modification indices). The SEMWISE approach also allows for the use of multi-group modeling for testing across-group measurement invariance and differences in parameters of interest. In addition, measurement error in the response variable can be accounted for. This is important as in many applications the response variable is latent (e.g., intention, attraction), so it may be desirable to use multiple indicators. Finally, the SEMWISE model can account for method effects, for instance by specifying a method factor that captures individual differences in rating scale use.

Our target audience includes researchers who (plan to) use policy capturing studies in organizational research but were not previously aware of the fact that they could analyze their data using SEM, as well as researchers who (plan to) use SEM but were not previously aware of its potential to deal with policy capturing data. Although we have tried to make this exposition of SEMWISE self-contained, some experience with SEM is probably required to fully understand the discussion (see Kline 2016).

We will start with a simple illustrative example of a policy capturing study that introduces the SEMWISE approach in an intuitive way. Next, we will discuss the SEMWISE model in greater detail by first presenting the core model and then offering several model extensions. These extensions will both show how the SEMWISE approach can deal with the complexities that often arise in policy capturing studies and demonstrate several potential advantages that the SEMWISE approach offers in specific situations. The empirical part of the paper will present two applications that illustrate the method and highlight some of its 
strengths. The paper ends with a comparison of the SEMWISE and multi-level modeling (MLM) approaches and guidelines on how to implement SEMWISE in practical settings.

\section{Introductory Example}

As an illustration of a SEMWISE analysis of policy capturing data, consider the fictitious example depicted in Figure 1, where participants are asked to rate four potential teammates for a quiz and the teammates are defined in terms of low vs. high warmth and competence. The researcher wants to estimate the weights (called part-worth utilities or partworths in conjoint studies) that participants attach to teammate warmth and competence when rating the experimental profiles, where the attribute weights are inferred from the overall ratings of the profiles by decomposing the overall rating into the contribution of warmth and competence to a profile's overall rating. The way this is accomplished in the SEMWISE approach is different from the way in which it is done in the conventional regression framework, although the results are equivalent. Appendix A discusses the regression approach and its similarities and differences with the SEMWISE approach; here, we will focus on

\section{SEMWISE.}

In the SEMWISE approach, a Confirmatory Factor Analysis (CFA) is specified in which the ratings of the four profiles are used as four indicators of three latent factors: an intercept factor and weight factors for warmth and competence. In contrast to a conventional CFA, each indicator is allowed to load on multiple factors (since each profile is composed of multiple attributes), and the factor loadings are fixed at particular values in order to link the overall profile ratings to the attribute levels characterizing the profiles. For instance, assuming that effect coding is used (where -1 indicates a low and +1 a high level on an attribute), a teammate profile with low warmth and high competence will have a loading of -1 on the warmth weight factor and a loading of +1 on the competence weight factor. The latent factors 
are called weight factors because they are unobserved variables that represent the individually varying weights that respondents attach to the manipulated attributes of the profiles (i.e., warmth and competence) when rating potential teammate profiles. In addition to the warmth and competence weight factors, a latent intercept factor is included to model respondents' average responses to all the profiles. The four profiles have fixed loadings of one on the intercept factor.

In what follows, we will explain the SEMWISE specification more formally, starting with the core model and then discussing several extensions. In Appendix A and in the general discussion, we provide further details about the similarities and differences between MLM and SEMWISE. Specifically, Appendix A shows how to analyze the introductory example (Figure 1) in MLM vs. SEM, demonstrates how the models can be estimated in Mplus, and compares the results obtained with the two methods for a simulated data set. The example shows that the parameter estimates are the same for this basic policy capturing model, although the terminology differs.

\section{The SEMWISE Model}

\section{The core model}

Consider a set of stimuli defined by p binary attributes of interest. Each attribute can be absent or present (or low vs. high), and a stimulus is defined by a particular combination of attribute levels. Using effect coding, -1 indicates the absence and +1 the presence of an attribute. Alternatively, one could use dummy codes of 0 and +1 . The levels of a stimulus on the $\mathrm{p}$ attributes affect a dependent variable $y$, the response variable. Figure 2 displays a path diagram and the corresponding algebraic formulation of the model for a situation where $\mathrm{p}=3$, which (in the case of a full factorial design) results in 8 alternative stimuli representing all possible combinations of the three binary attributes $\left(2^{3}\right)$. 
It is apparent that the model depicted in Figure 2 is a factor model. Though not shown in Figure 2, the factor model includes means and intercept terms (for an introduction to modeling means in SEM, see Ployhart \& Oswald 2004). In contrast to the traditional factor model, all loadings are fixed at values of -1 or +1 (assuming effect-coding). Also, each observed variable is allowed to load on multiple factors because each profile represents a combination of attribute levels. For example, $\mathrm{y}_{1}$ is the response to a profile in which all three attributes are present. The loading matrix links participants' observed responses to each profile ( $y_{1}$ to $\left.y_{8}\right)$ to four latent factors: an intercept factor $\left(\eta_{0}\right)$ and three latent weight factors $\left(\eta_{1}\right.$ to $\left.\eta_{3}\right)$. The intercept factor has a unit loading on each observed variable and represents the average response per individual respondent; it captures true differences in average preference but also differences in scale use (i.e., rater bias). The latent weight factors represent individual differences in the weights assigned to the three attributes characterizing each profile, and these weight factors are of primary interest in policy capturing studies. Specifically, within the SEMWISE model, the means, variances and covariances of the weight factors are estimated and the weight factors can be related to other constructs, as explained below. It is therefore not necessary to explicitly compute the individual weights (i.e., factor scores).

The latent means of the weight factors $\eta_{1}$ to $\eta_{3}$ represent the average (positive or negative) contribution of each attribute (depending on whether the attribute is present or absent, or high or low) to the overall ratings across all individuals in the sample (e.g., how much high warmth adds to the overall ratings of profiles on average). The latent mean of the intercept factor $\eta_{0}$ captures the average response across all respondents and stimuli (and will typically be of little interest).

The variances of the weight factors represent the extent to which the individuallyvarying weights fluctuate about the mean of each weight factor (e.g., how much the weights attached to warmth vary across individuals); the covariances of the weight factors represent 
the extent to which the weights of different factors correlate across individuals (e.g., whether individuals who value high warmth also tend to value high competence). If the variances of the latent weight factors $\eta_{1}$ to $\eta_{3}$ are close to zero, this means that the weights are homogeneous across respondents and that the random-effect specification (in which the factor scores for a given attribute are allowed to vary across respondents) reduces to a fixed-effect specification (in which the factor scores are the same for each respondent). In the same vein, a fixed intercept specification is obtained by setting the variance of the intercept factor $\eta_{0}$ to zero; this means that the average rating across all profiles is the same for each respondent. Of course, if the factor variances are zero, the covariances are also zero. The question of whether the effects of $\eta_{0}$ to $\eta_{3}$ ought to be specified as random or fixed effects can be addressed based on extant theory, by evaluating the confidence intervals of the variance terms in question and/or by testing model constraints on the variance terms (i.e., evaluating the deterioration in model fit when fixing the variance terms to zero by means of chi-square difference testing).

Finally, individual differences in stimulus-specific y-scores are represented by the (error) terms $\varepsilon_{1}$ to $\varepsilon_{8}$, which are assumed to have a mean of zero. These error terms can be interpreted as deviations between the ratings of a profile predicted by the model and the ratings actually observed, and the variances of the errors are generally freely estimated in the SEMWISE model.

Before the specified model can be estimated, the identification of the model has to be ascertained (see Bollen, 1989; Mulaik, 2009). General identification rules that apply to any type of SEMWISE model do not exist. However, a necessary condition for identification is that the number of unknown model parameters does not exceed the number of unique variances, covariances, and means of the observed variables (i.e., that the degrees of freedom is nonnegative). For example, for the model in Figure 2, there are 36 unique elements in the variance-covariance matrix of the observed variables and 8 observed means, and since the 
number of unknown model parameters is 22 (10 factor variances and covariances, 8 error variances, and 4 factor means), the model has 22 degrees of freedom (44 minus 22). Although a model that satisfies the necessary identification condition is not necessarily globally identified, the model in Figure 2 can be shown to be identified from first principles. One characteristic of SEMWISE models that facilitates identification is that the factor loadings are fixed to particular values. In general, policy capturing models similar to that in Figure 2 will be identified, but for more complicated models (see below), identification has to be evaluated on a case-by-case basis.

Once the specified model has been estimated, it can be evaluated in more detail as follows. First, the overall fit of the model should be examined in order to assess to what extent the model is consistent with the data (although a well-fitting model does not preclude the possibility that other models fit the data equally well). The fit indices and suggested cutoff values usually applied in confirmatory factor analysis can be used here (Cheung \& Rensvold, 2001; Lance, Butts, \& Michels, 2006). Second, even when the global fit is acceptable, it is instructive to check indices of local misfit (residuals and modification indices) in order to identify specific problematic model constraints. Third, researchers need to verify that the estimated parameters are meaningful (e.g., that there are no negative variance estimates, which can occur in more complicated models incorporating interactions among the attributes). Finally, if the previous conditions hold, the parameter estimates can be interpreted and the explained variance in each indicator can be examined to verify that respondents' ratings are mainly driven by the manipulated attributes. The weight factor means, variances and covariances can also be evaluated and, if necessary, further model restrictions and/or extensions may be considered (e.g., weight factor variances can be restricted to zero).

In what follows, we discuss several model extensions that broaden the applicability of SEMWISE to other designs encountered in policy capturing studies and capitalize on the 
modeling capabilities offered by SEM. The extensions are not exhaustive and can be used in combination. The Web Appendix provides Mplus syntax for generating data and Mplus, Lavaan and Lisrel syntax for analyzing the data consistent with the core model (see Figure 2) and most of the extensions discussed next.

\section{Model Extensions}

\section{Model extension 1: Attributes with more than two levels}

Some independent variables of interest have more than two categories or levels. Web Appendix Example 2 presents syntax for policy capturing with non-binary attributes. Independent variables can be metric or categorical. For metric independent variables, the relation between the independent variable and the response variable y can be linear or nonlinear. Linearity can be modeled by using loadings with equal intervals, such as 1,2 , and 3 if there are three equally spaced levels of the manipulated attribute (e.g., price levels of $\$ 1$, \$2, and \$3). Linearity can also be imposed in instances where an independent variable takes on different levels (e.g., low, medium, high) that can be reasonably assumed to be equidistant (so-called allocated coding; see Aiman-Smith et al., 2002). In order to model a nonlinear relationship, a combination of fixed and freely estimated weights can be used. For example, if respondents rate applicants with varying grades (e.g., satisfaction, distinction, and high distinction), satisfaction could get a weight of -1 , high distinction a weight of +1 , and the weight of distinction could be freely estimated. Finally, if the levels of an attribute are unordered and categorical, (k-1) factors are needed to represent the information in the $\mathrm{k}$ categories. For example, if the design includes products from three countries of origin (e.g., the U.S., Germany, and China), two weight factors are needed and the loadings for the first (second) weight factor could be specified as $+1,0$, and $-1(0,+1$, and -1$)$ for products from the U.S., Germany, and China, respectively. 


\section{Model extension 2: Interactions between the attributes}

So far we have assumed the responses are only functions of the main effects of the experimentally manipulated attributes. One can also model interactions between attributes by adding latent factors that represent combinations of attribute levels of different factors. Operationally, the loadings for an interaction factor equal the product of the loadings of its constituent effects (as in regression analysis). For example, if the interaction between attributes 1 and 2 were added to the model in Figure 2, the loadings would be $+1,-1,-1,+1$, $+1,-1,-1,+1$, and an additional weight factor $\eta_{4}$ would be added to the model. Three-way or even higher-order interactions can also be included by multiplying the loadings of the factors involved in the interaction. Of course, the interpretation of interactions becomes increasingly complex as more higher-order terms are added to the model. In addition, since such models have fewer degrees of freedom, various estimation problems can occur in models containing high-order interactions (e.g., negative error variances).

Some results in terms of the number of parameters to be estimated for different designs are summarized in Table 2. This table also provides important input for assessing model identification, because the degrees of freedom in an identified model cannot be negative. If all possible main and interaction effects are included in the model, the model is saturated and can exactly reproduce the data (without testing anything), so that the error variances can be fixed at zero. If mean, variance and covariance parameters associated with higher order interaction factors are near zero, then simpler models containing fewer interactions should be entertained. For reasons of parsimony, we will henceforth focus on models with only main effects. However, in some research contexts, interactions are of theoretical interest, in which case they should be included in the model. Furthermore, if the fit of the model is poor and if the main effects explain only a small portion of the variance in observed responses, it may be necessary to consider interaction factors. 


\section{Model extension 3: Multiple indicators for the dependent variable}

Sometimes, the response variable in policy capturing experiments is best conceptualized as a latent variable, so researchers may want to use multiple indicators to measure it (e.g., Aiman-Smith, Bauer, \& Cable 2001). Using multiple indicators for the profile ratings makes it possible to better account for measurement error in the ratings, so that attenuation due to measurement error can be corrected for within the model. Also, the use of multiple measurement methods enables researchers to separate method variance from substantive variance, in line with the multitrait-multimethod tradition (Lance, Noble, \& Scullen, 2002).

This approach is illustrated in Figure 3 for a model with four main effects and eight stimuli; the Web Appendix (see example 3) provides the corresponding syntax (including parameter restrictions that make this model identified). As an example, respondents could rate the attractiveness of experimentally manipulated teammate descriptions on a five-point scale (variables $\mathrm{y}_{1}$ to $\mathrm{y}_{8}$ in Figure 3 ) as well as by means of a continuous slider scale (variables $\mathrm{z}_{1}$ to $\mathrm{Z}_{8}$ in Figure 3). As can be seen in Figure 3, the two ratings for each profile together load on a first-order factor (the $\eta$-factors). The first-order factors then act as latent indicators of the weight factors (the $\xi$ factors), which are now second-order factors, but otherwise the interpretation is the same as in a single-indicator model. At the same time, as can be seen on the right-hand side of Figure 3, all ratings using the same format also load on a methodspecific intercept factor (see $\mathrm{M}_{\mathrm{y}}$ and $\mathrm{M}_{\mathrm{z}}$ in Figure 3), which we will simply refer to as method factors. A method factor is generally defined as a latent variable assumed to contribute to the variability of a set of observed response variables that share a common method (Weijters, Baumgartner, \& Schillewaert, 2013). The method factors capture the average rating that each respondent gives to the entire set of profiles in a given scale format; this includes the average preference as well as individual format-specific scale use (i.e., rater bias) (Maydeu-Olivares \& 
Coffman, 2006). Integrating a multi-indicator measurement model into the policy capturing model is something that cannot be done in the standard MLM framework.

\section{Model extension 4: Adding antecedents and consequences of the weight factors}

A common type of research question in policy capturing research pertains to explaining individual differences in the weight or importance of attributes (e.g., Grégoire \& Shepherd 2012). In MLM the consideration of potential determinants of individually varying weights results in cross-level interactions between the experimentally manipulated attributes characterizing each profile (at level 1) and the individual difference variable of interest (at level 2). In SEMWISE, the effects of individual difference variables are modeled as antecedents of the weight factors. Referring back to our introductory example (see Figure 1), to test whether respondents' need for achievement affects the weight they attach to warmth and competence in evaluating potential teammates, respondents' need for achievement could be added as an antecedent of the weight factors for warmth and competence.

A similar approach can be used for nominal antecedents. For instance, in our introductory example (Figure 1), gender might be hypothesized to influence the weights of warmth and competence in evaluating potential teammates. In SEMWISE, this could be analyzed by adding a dummy variable for gender as an observed antecedent of the weight factors (see Figure 4a and Example 4a in the Web Appendix). The regression weight linking the dummy variable to the weight factors would represent the gender difference in the extent to which warmth and competence affect potential teammate evaluations.

A SEMWISE model can also specify the weight factors as antecedents of subsequent outcome variables (see Figure 4b). For instance, a researcher could study entrepreneurs' risk seeking tendencies in a policy capturing design and model the weight factors as antecedents of later entrepreneurial activity in risky markets. The weight factors can also act as mediating variables (see Example 5 in the Web Appendix). There is no direct equivalent of this 
approach in an MLM framework, although as an indirect approximation, one could save individual estimates of regression coefficients and use them in subsequent analyses.

\section{Model extension 5: Multiple groups}

Observed categorical variables can also be used as grouping variables. For this extension (multiple group analysis) there is no counterpart in the standard MLM framework. For instance, instead of using gender as an antecedent, respondents' gender could be used as a grouping variable, and the same SEMWISE model could be simultaneously estimated for two samples differing in gender (see Figure $4 c$ and example $4 b$ in the Web Appendix). In this setup, the between-group mean difference in the SEMWISE factors would capture the gender difference in the extent to which a specific attribute (e.g., warmth) affects teammate evaluations. But in addition, one could also test for between-group differences in the weight factor variances, which would indicate whether one group is more or less heterogeneous in terms of the importance assigned to a given attribute.

When combining multi-group specifications with the consideration of antecedents and consequences, the grouping variable can also be studied as a moderator of the relationship between the weight factors and the antecedents or consequences. For instance, one could investigate whether the effect of need for achievement (the antecedent) on the weights of warmth and competence in teammate choice is moderated by respondent gender (the grouping variable).

\section{Overview of empirical applications}

To illustrate the SEMWISE approach, we now report two empirical applications. First, using policy capturing data on potential teammate preferences, we illustrate the core SEMWISE model (cf. Figure 2), as well as an extension with an observed antecedent used either as a covariate (cf. Figure 4a) or as a grouping variable (cf. Figure 4c). Second, we use policy capturing data on organizational attractiveness to illustrate models in which the 
response variable is measured with multiple indicators (cf. Figure 3) and the latent weight factors are related to latent antecedents.

\section{Empirical application 1}

The first empirical application is an illustrative policy capturing study concerning evaluations of potential teammates. The approach was inspired by Caruso, Rahnev, and Banaji (2009). In the current study, student participants (men and women) rated potential teammates (all women) for a trivia game of math and science. Fictitious team member profiles were created that vary in terms of a clearly task-relevant attribute (competence, more specifically IQ), a possibly process-relevant attribute (perceived warmth), and an irrelevant attribute (facial attractiveness). When evaluating potential teammates, above average IQ is expected to be valued in a teammate because it increases the chances of winning (Caruso et al., 2009). Warmth, although offering less of a direct competitive advantage, could be functional in terms of facilitating trust and mutual coordination (Cuddy, Glick, \& Beninger, 2011). Facial attractiveness is presumably irrelevant to the task at hand, but it is plausible that mate selection preferences for attractive faces developed through evolution will influence men's (although possibly not women's) teammate preferences (Fink \& Penton-Voak, 2002).

\section{Method}

We constructed eight profiles using a full factorial design in which we experimentally manipulated attractiveness (below vs. above average), warmth (low vs. high) and competence (average vs. high IQ). All experimental profiles depicted white women. For each experimental profile, we constructed a profile description by randomly sampling a female name, a (low vs. high) warmth description, and an (average vs. high) IQ level from the appropriate pool of elements (e.g., an attractive picture with a low-warmth description and high IQ level), using 
the form "[Picture]. [Name] is described by her friends as [warmth level]. She has an IQ of [IQ level].” The specific levels were sampled from the sets of elements shown in Table 3.

Thus, each respondent saw a series of different profiles for which the name, picture and warmth description were never identical (IQ levels could coincidentally replicate, but not systematically so). This approach was intended to make the task less transparent and more involving, and to present participants with stimuli sampled from a broader domain. As a potential side-effect, it is likely to increase random variation in responses, but the SEMWISE model explicitly accounts for this (in the residual terms for each of the eight responses).

The experimental profiles were shown one per page, in randomized order. We added a replication profile to test participants' consistency; this ninth profile was the same for all respondents (except for the name), but again did not verbally or visually replicate any of the other profiles and corresponded to an above average attractiveness, low warmth, high competence profile. The correlation between the ratings for the two replicated profiles was $r=.60$, which suggests reasonable consistency (especially in light of the random sampling of the elements making up the profile).

Participants rated each profile on a visual scale using stars with a continuous range from 0 to 5. To get acquainted with the task, participants had to give three ratings in line with the following instructions: "To get acquainted with the question format, please rate the profiles below in line with the instructions (by selecting a score from zero to five stars): (1) Person A has all the rights skills and the right personality. Give this profile a very high score. (2) Person B is average. Give this profile an intermediate score. (3) Person C does not have the right skills and not the kind of personality you are looking for. Give this profile a very low score." To be able to proceed to the next page, respondents had to give a rating above 3 for person $\mathrm{A}$ and a rating below 3 for person $\mathrm{C}$. 
We collected data from students at a U.S. university, retaining for analysis only those respondents who indicated English as their native language (because the personality descriptions were verbally demanding) and White/Caucasian as their race (to avoid potential confounding effects of respondent sex and race). In our sample, $\mathrm{N}=260$, ages range from 19 to 21 years $(\mathrm{M}=19.4, \mathrm{SD}=.55)$, and $46.9 \%$ of participants are women. Appendix $\mathrm{B}$ reports the correlation matrix.

\section{Results}

The design of this study corresponds to the core SEMWISE model presented in Figure 2. The baseline model shows acceptable fit to the data $\left(\chi^{2}(22)=41.864, p=.006\right.$, RMSEA $=$ $.059, \mathrm{CFI}=.972, \mathrm{TLI}=.965, \mathrm{SRMR}=.058)$. The percentage of explained variance in the eight profile ratings ranges from .52 to .63 (all $\mathrm{p}<.001$ ). The weight factor means and variance terms (and their associated standard errors) are reported in Table 4 and are all significantly different from zero. Correlations between the weight factors are all nonsignificant, with the exception of the correlation between warmth and competence $(r=-.29, p$ $<.01)$. These results indicate that competence, warmth and facial attractiveness all contribute (positively) to teammate preferences, despite the irrelevance of facial attractiveness for the task at hand. Also, there is individual variation in the weight assigned to each of the attributes. Finally, participants who attach more weight to competence tend to attach less weight to warmth.

Adding covariates as a determinant of the weight factors is one way of trying to better understand the individual variation in the weight factors. We estimate a SEMWISE model using participants' gender (FEMALE; $0=$ male, $1=$ female) as a covariate. This model corresponds to the SEMWISE extension with an observed antecedent used as a covariate (cf. Figure 4a) and it shows acceptable fit to the data $\left(\chi^{2}(26)=43.915, \mathrm{p}=.015, \mathrm{RMSEA}=.051\right.$, $\mathrm{CFI}=.975, \mathrm{TLI}=.965, \mathrm{SRMR}=.053)$. The effect of FEMALE on the attractiveness weight 
factor is negative but not statistically significant $(\mathrm{B}=-.049, \mathrm{SE}=.030, \mathrm{t}=-1.625, \mathrm{p}=.104)$. None of the other weight factors is significantly related to FEMALE either (all $\mathrm{p}>.40$ ). Furthermore, when controlling for participants' gender, the correlation between the warmth and competence weight factors remains significantly negative $(r=-.29, p<.01)$.

To further investigate gender differences in team member evaluation, we run a twogroup SEMWISE model, using participants' gender as the grouping variable. This approach corresponds to the model extension depicted in Figure 4c. The model shows acceptable fit to the data $\left(\chi^{2}(44)=67.156, p=.014\right.$, RMSEA $=.064$, CFI $=.968$, TLI $=.960$, SRMR $\left.=.067\right)$. The parameter estimates are reported in Table 5.

This analysis yields some additional results that cannot be obtained from the previous model (where FEMALE was a covariate, not a grouping variable) nor from a multilevel analysis. In general, gender differences are small, but some differences between men and women are of note. First, the mean of the attractiveness weight factor is significant among men $(\mathrm{M}=.079, \mathrm{t}=3.424, \mathrm{p}=.001)$ but not among women $(\mathrm{M}=.034, \mathrm{t}=1.753, \mathrm{p}=.080)$; however, the difference between the two means is not significant $(t=1.494, p=0.135)$. In addition, the variance of the attractiveness weight factor is significant among men (Var. = $.025, \mathrm{p}<.01)$ but not among women $($ Var. $=.002, \mathrm{p}=.730)$; this difference between the two variances is statistically significant $(\mathrm{t}=2.051, \mathrm{p}=.040)$. In sum, for male participants facial attractiveness has a small but statistically significant effect on teammate preferences on average, although there are significant individual differences in the magnitude of this effect across men. In contrast, for female participants facial attractiveness does not have a significant effect on average, and there is little variation in this effect across women. We also find that the negative correlation between the weight of warmth and the weight of competence found in the sample as a whole only holds for men $(r=-.45, \mathrm{p}=.001)$, not for women $(\mathrm{r}=-.14, \mathrm{p}=.298)$, although the difference in the magnitude of the correlation is not 
significant (Wald $\chi^{2}(1)=3.218, \mathrm{p}=.073$ ). This finding suggests that men may have a stronger tendency to focus either on warmth or on competence (but not both) when evaluating potential female teammates.

\section{Empirical application 2}

For the second empirical application, we collected data using multiple indicators for the response variable. Similar to Aiman-Smith et al. (2001), we use policy capturing to examine the relative influence of four factors (two job factors of pay and promotion, and two organizational image factors of lay-off policy and ecological rating) on potential job recruits' attraction to an organization.

\section{Method}

We designed company profile descriptions by manipulating the same four attributes as Aiman-Smith et al. (2001), but specifying only two levels for each attribute. Specifically we generated eight profiles in a fractional factorial design as reported in Table 6. Respondents judged organizational attractiveness based on each profile using two items: (a) 'To what extent would you like to work for this company?' (rated by manipulating a sliding bar linked to a smiley face with five levels ranging from $1=$ low to $5=$ high) and (b) 'How attractive do you find this company?' (rated by means of a continuous sliding scale marked by five stars with scores ranging from zero to five). These formats were chosen because they are visually attractive and engaging for respondents, and they also are different from the Likert scales we used for other variables. The profiles were presented in randomized order to all respondents. The eighth profile was presented twice (and included in the randomized profile sequence) to test the internal consistency of the policy capturing responses. The correlation between the responses to the two replicated profiles (using a two-factor model with two items per factor 
and specifying unit loadings) was $r=.66(p<.001)$, which suggests that respondents' ratings were reasonably consistent.

Respondents also answered a six-item scale on Environmental Concern (EC), adapted from Catlin and Wang (2012), in which half the items were reverse-scored: I would describe myself as environmentally responsible; I consider the potential environmental impact of my actions when making many of my decisions; I am concerned about wasting the resources of our planet; My habits are not affected by environmental concerns (reversed); Environmentally friendly actions are too inconvenient for me (reversed); I'm not particularly bothered by worries about our environment (reversed). Finally, a shortened 10-item version of the Impression Management (IM) scale (Paulhus, 1991) was included to control for socially desirable responding. This scale also consists of a mix of reversed and non-reversed items.

We collected data from students at a U.S. university. In our sample, $\mathrm{N}=276$, age ranges from 18 to $36(\mathrm{M}=19.6, \mathrm{SD}=1.9)$, and $44.9 \%$ are women. Appendix $\mathrm{C}$ reports the correlation matrix of all study variables.

\section{Results}

To illustrate the integrated use of a measurement model with rating scale format method factors, we run a SEMWISE model corresponding to the one shown in Figure 3. For each of the eight focal profiles (i.e., not including the replicated profile), we specify one factor with two indicators. For each of these eight factors, the first indicator has a unit loading on the underlying factor for purposes of identification; the loading of the second indicator is constrained to be equal across all eight factors (as there is no reason why the same scale should be less reliable for certain profiles than others). These first-order factors ( $\eta_{1}$ to $\left.\eta_{8}\right)$ serve as the SEMWISE indicator variables of the second-order weight factors ( $\xi_{1}$ to $\left.\xi_{4}\right)$ with loadings fixed depending on the attribute levels contained in a profile, as listed in Table 6 . To capture scale usage effects for the two scale formats, we specify two method factors, $\mathrm{M}_{\mathrm{y}}$ and 
$\mathrm{M}_{\mathrm{z}}$, one for each scale format, which freely correlate with each other and with the weight factors $\xi_{1}$ to $\xi_{4}$ (correlations between factors are not shown in Figure 3 for readability). The indicator intercepts are fixed to zero, but the means of the method factors are freely estimated (as are the means of the weight factors $\xi_{1}$ to $\xi_{4}$ ). The method factor means represent the average scale response for each of the two formats and they capture both individuals' scale usage (rater bias) and average attractiveness perceptions. The resulting model shows acceptable fit to the data $\left(\chi^{2}(100)=144.174, \mathrm{p}=.003, \mathrm{RMSEA}=.040, \mathrm{CFI}=.983, \mathrm{TLI}=\right.$ $.980, \mathrm{SRMR}=.047)$. Percentage of explained variance ranges from .75 to .88 for the $y$ indicators and from .76 to .87 for the z-indicators. Means, variances, and correlations of the four weight factors are reported in Table 6 . All weight factors have statistically significant means and variances, indicating that compensation, promotion, layoff policy and a company's environmental concern all affect organizational attractiveness, although the two job factors (pay and promotion) are on average more important than are the two organizational image factors (relating to lay-off policy and a firm's ecological rating). Also, respondents who attach more weight to promotion tend to attach less weight to a company's layoff policies $(r=-.40$, $\mathrm{p}<.05)$ and environmental concern $(\mathrm{r}=-.32, \mathrm{p}<.05)$. The means for the two method factors are $\mathrm{M}_{\mathrm{y}}=2.752(\mathrm{SE}=.027)$ and $\mathrm{M}_{\mathrm{z}}=2.309(\mathrm{SE}=.035)$, with a correlation of $\mathrm{r}=.765(\mathrm{SE}=$ .034). The method factors do not show significant correlations with the weight factors, with the exception of a correlation between $\mathrm{M}_{\mathrm{y}}$ and the layoff policy factor, $\mathrm{r}=.265(\mathrm{SE}=.125, \mathrm{p}$ $=.034)$.

Next, we add two latent variables: EC is included as an antecedent of the SEMWISE weight factors, and IM is added as a control variable. The goal of this extension is twofold. First, it illustrates how the SEMWISE model can be easily integrated into a broader nomological network. Second, it allows us to validate the assumption that policy capturing designs (especially the environmental concern weight factor) are less vulnerable to social 
desirability than self-reports based on direct questioning (in this case, the EC scale)

(Tomassetti, Dalal, \& Kaplan, 2016). This is especially relevant for environmental concern, which, because of its moral connotations, is likely to be susceptible to the influence of socially desirable responding (Jones \& Willness, 2013).

We start with a preliminary confirmatory factor analysis (CFA) of the latent covariates Environmental Concern (EC) and Impression Management (IM). Both scales contain reversed items (reversed items were not reverse-scored), and we include a method factor $\mathrm{M}_{\mathrm{x}}$ to control for individual variation in scale usage (Maydeu-Olivares \& Coffman, 2006; Weijters et al., 2013); all indicators, irrespective of wording direction, have unit loadings on the method factor, whereas the loadings on the substantive factors are freely estimated (so the loadings for regular and reversed items are expected to have opposite signs). For the sake of parsimony and model fit (and since our focus is not on the measurement parameters of this scale but the structural estimates), we use four item parcels for IM (two parcels based on the reversed items and two based on the non-reversed items; Rhemtulla, 2016). The resulting CFA model (with parcels for IM and with a method factor) fits the data well $\left(\chi^{2}(33)=42.645\right.$, RMSEA $=.033$, $\mathrm{CFI}=.985, \mathrm{TLI}=.980, \mathrm{SRMR}=.042)$. The method factor has a variance term significantly different from zero $(\mathrm{p}<.001)$, and omitting the method factor results in a significant deterioration in fit $\left(\Delta \chi^{2}(1)=27.61, \mathrm{p}<.001\right)$.

Next, we specify EC and IM as antecedents of the weight factors, as shown in Figure 5. For readability, the figure does not contain factor correlations, but the following sets of factors are allowed to covary: (a) the three method factors $\mathrm{M}_{\mathrm{x}}, \mathrm{M}_{\mathrm{y}}$, and $\mathrm{M}_{\mathrm{z}}$, where the only significant correlation is the one already found in the core model, between $\mathrm{M}_{\mathrm{y}}$ and $\mathrm{M}_{\mathrm{z}}$ $(\mathrm{r}=.77, \mathrm{SE}=.03, \mathrm{p}<.001) ;(\mathrm{b})$ the weight factors compensation $(\mathrm{COMP})$, promotion (PROMO), Layoff policy (LAYOFF) and the company's environmental concern (CEC) (as already discussed in the core model); and (c) EC and IM, which have a significant positive 
correlation of $\mathrm{r}=.18(\mathrm{SE}=.08, \mathrm{p}=.033)$. The resulting model fits the data well: $\chi^{2}(280)=$ $299.744, \mathrm{p}=.200 ; \mathrm{RMSEA}=.016, \mathrm{CFI}=.994, \mathrm{TLI}=.993, \mathrm{SRMR}=.047$. The only significant regression weight linking the SEMWISE weight factors (COMP, PROMO, LAYOFF and CEC) to EC and IM is the one between EC and CEC, with standardized $\mathrm{B}=.83(\mathrm{SE}=.07, \mathrm{p}<.001)$. Thus, those who are high on self-rated environmental concern actually assigned a greater weight to a company's environmental record when indicating their attraction to a company. Interestingly, CEC is not significantly related to IM (Standardized $\mathrm{B}=-.13, \mathrm{SE}=.10, \mathrm{p}=.197)$. The results provide support for the convergent validity of the CEC factor, as it is strongly related to individuals' EC, although-interestingly - the CEC weight factor is less affected by IM than is the EC scale. This is consistent with the recent finding by Tomassetti et al. (2016) that policy capturing is more resistant to socially desirable responding than traditional self-report techniques.

\section{Discussion}

In the current paper, we introduced SEMWISE (Structural Equation Modeling for Within-Subject Experiments) and demonstrated how it can be used for analyzing policy capturing data. To give credit where credit is due, we want to stress that the proposed approach constitutes a specific application of the more general principle posited by Curran (2003), who stated that "[a]ny two-level linear multilevel model can be estimated as a structural equation model given that this is essentially a data management problem" (p. 557). This equivalence between MLM and SEM is commonly capitalized on in latent curve modeling, where repeated observations over time are nested within individuals and where the use of SEM is well established (Feingold, 2009). Whereas MLM uses time as an independent variable in such instances, latent curve models based on SEM incorporate time as fixed values within the factor loading matrix (Curran, 2003). Within-subject experimental designs, 
including policy capturing designs, constitute a similar data structure, in that repeated measurements are related to fixed experimental variables (the manipulated attributes). Thus, here too the fixed values for the experimental manipulations can either be included as independent variables in a multilevel model or as fixed values of the factor loading matrix in an SEM.

Two important questions need some further elaboration and will be addressed in more detail now. First, what are some practical guidelines for implementing SEMWISE? Second, when should researchers stick with Multi-Level Modeling (MLM), and when might they want to opt for SEMWISE instead?

\section{Application guidelines}

With regard to the first question, the empirical applications can serve as examples for researchers who want to apply a similar approach. More generally, we suggest the stepwise approach shown in Figure 6. As a first step, we propose starting with the core model (without antecedents or outcomes) while accounting for multi-group and/or multi-indicator data from the start and deciding how to specify the measurement parameters of the model (i.e., loadings, second-order factor structure if applicable, and residual variances). In a second step, the resulting model needs to be carefully evaluated, returning to step one if necessary. A third step involves including interaction terms if required. In a fourth step, the variances of the weight factors are evaluated, so that antecedents, correlates and/or outcomes can be added for those weight factors that show significant variation between individuals, and if applicable, parameters are tested for across-group invariance. The final step involves interpretation and reporting.

We did not encounter issues with model non-convergence or improper estimates, neither in our empirical applications nor our simulations (see Web Appendix). If researchers do run into such problems, we recommend two things. First, model identification needs to be 
verified. The models presented in the current paper are all identified and the Web Appendix provides syntax including constraints required for identification. For a more detailed discussion of model identification, we refer to chapter six in Mulaik (2009). Second, if the model is identified but still results in improper estimates, we recommend using Bayesian estimation with minimally informative prior distributions, which strongly facilatates convergence without inducing bias, as recently demonstrated by Helm et al. (2017).

\section{Choosing between MLM and SEMWISE}

The primary objective of this paper is to demonstrate the potential of the proposed SEMWISE approach. To be clear, the goal is not to question the usefulness of MLM for analyzing policy capturing data. Table 7 summarizes the main similarities and differences between MLM and the SEMWISE approach as applied to policy capturing. In common policy capturing studies, the SEMWISE and MLM approaches will lead to the same results. To demonstrate this equivalence, Appendix A contains syntax for generating a dataset in accordance with the stylized policy capturing example considered earlier (Figure 1) and for analyzing the data using both SEM and MLM. The resulting parameter estimates are identical. However, the setup of the data file, the specification of the model, and the labeling of the parameters are all different (see Appendix A). A prime consideration in choosing between MLM and SEMWISE therefore will often relate to a researcher's familiarity with and/or preference for MLM versus SEM. As a rule, the MLM and SEM approaches for analyzing policy capturing data are equivalent for data from a single group of respondents, using a single indicator per profile, where no outcomes are related to the weights. Since such instances are quite common, MLM is a good choice for many policy capturing studies. For instance, MLM is well suited for analyzing the examples from the literature mentioned in the introduction and in Table 1. That being said, SEMWISE offers some interesting modeling possibilities that future policy capturing researchers may want to capitalize on, primarily 
related to (a) measurement, (b) model evaluation and comparison, and (c) the types of hypotheses that can be tested.

Related to measurement, research on organizational and managerial behavior has long been characterized by a strong emphasis on scale construction and measure validation, in which Confirmatory Factor Analysis and Structural Equation Modeling have played an important role (Edwards, 2001; Hinkin, 1998). The SEMWISE approach can facilitate the same measurement rigor in policy capturing research. In the core model (Figure 2), each profile rating has a residual variance term, which captures a mix of random measurement error, method effects, and profile-specific variance. The residual variance term specification is flexible, in that residual variances can be allowed to differ across profiles and residual correlations could be included if there are reasons for doing so, for instance if profiles are shown in a fixed sequence and order effects occur. A more sophisticated measurement model is offered by the multi-indicator model (Figure 3), where residual terms are estimated at two levels: The indicators (the profile ratings) have residual terms that capture the variance that remains (presumably consisting mostly of random error variance) after accounting for profilespecific and method-specific effects, and the first-order factors $\left(\eta_{1}\right.$ through $\eta_{8}$ in Figure 3$)$ have residual terms that capture variance not accounted for by the weight factors (after accounting for scale-specific method effects).

Second, in terms of model evaluation and comparison, SEM offers a well-established framework for evaluating model fit (Mulaik, 2009). This is helpful for comparing alternative models (e.g., models containing more or less restrictive residual variance specifications), but also when evaluating a single model in isolation, where a good model fit suggests that the observed data are consistent with a given theoretical model (even though it is possible that other models may fit even better or equally well). In the case of policy capturing studies, good model fit supports the assumption that the ratings are indeed driven by the latent weight 
factors. Problematic model fit indicates that somehow the model is not in line with the observed data, which (among other things) can be due to non-modeled correlations (e.g., two profiles may have unintended similarities, or presentation order effects may be at play), or which may suggest the need to reconsider parameter restrictions (e.g., to allow for nonlinearities in the effect of an attribute on profile ratings) or include additional terms in the model (e.g., adding interaction terms to a main-effects only model).

Third, researchers may have specific modeling needs that can only be handled in a SEMWISE framework. Two important extensions in this regard are multi-group modeling and the possibility to specify more comprehensive models that include antecedents and/or outcomes of weight factors. Variance-covariance structures can be compared across multiple samples (e.g., men vs. women, across countries or organizations, etc.) by using multi-group SEM (Vandenberg \& Lance, 2000). In our first empirical example, for instance, we found that the attractiveness weight factor showed greater variance among male participants compared to female participants. This illustrates how the approach facilitates across-group comparisons of model parameters other than weight factor means.

Another interesting modeling opportunity is that by adding antecedents, correlates and outcomes of the weight factors, the weight factors can be embedded in a broader nomological network with other latent and/or observed variables. In this way, policy capturing weight factors could be used as mediating variables. To illustrate, consider a variation on the first empirical application, where men vs. women (the antecedent) would be compared in terms of the weight they attach to the physical attractiveness of candidates (i.e., the mediating weight factors). These weights could then act as antecedents of outcomes of interest, such as biased selection choices or evaluations. Similarly, entrepreneurs from different countries or from different industries (antecedent) might attach different weights to social control or other 
characteristics of strategic alliances (i.e., the mediating weight factors), which could in turn predict engagement in alliances differing in duration and goal realization (outcome).

In sum, SEMWISE may be preferable to multilevel analysis in some situations, including the following: (a) the dependent variable is latent (e.g., attractiveness, performance ratings) and researchers want to correct for measurement error and method variance in the data (e.g., related to the use of particular rating scale formats); (b) researchers want to carefully evaluate their model specification and/or examine alternative model specifications in terms of fit with the data; and (c) the model of interest entails across-group comparisons of parameters other than weight means (e.g., variance terms of weight factors) and/or the examination of nomological networks involving other constructs, in which the weight factors serve as antecedents or mediating variables of outcomes of interest.

\section{Concluding Remarks}

SEMWISE is a method for analyzing data collected in a policy capturing study. The design of the study will usually not require adaptation, and we refer to excellent review-based tutorials on how to set up policy capturing studies, including Aiman-Smith et al. (2002), Karren and Barringer (2002) and Aguinis and Bradley (2014). Researchers planning to use the SEMWISE approach may want to spend some extra thought on the use of multiple indicators for the profile ratings. If researchers want to explicitly model method factors, it is recommended that they measure the response variable with several different formats. In the second empirical example, we used two indicators (a smiley face format and a five-star rating). Future research may want to explore which types of formats perform best in terms of respondent motivation and data quality.

In certain types of policy capturing studies, researchers who plan to use SEMWISE may deem it beneficial to limit the number of profiles presented to respondents to reduce respondent burden and leave more opportunity for measuring other variables, specifically 
when the goal is to study a broader nomological network surrounding the weight factors. In such instances, we recommend that researchers first consult in more detail sources that discuss tradeoffs related to presenting a small versus a large numbers of profiles (AimanSmith et al., 2002; Graham \& Cable, 2001; Sauer, Auspurg, Hinz, \& Liebig, 2011). Advanced users could adapt the syntax provided in Appendix A for running Monte Carlo power analyses to determine the appropriate number of profiles and/or respondents (Muthén \& Muthén, 2002).

The focus of the current paper is on using SEM for analyzing policy capturing data, but—as implied by the name given to the approach—its applicability is broader. To illustrate, some of the studies reported in Table 1 are not policy capturing studies in the narrow sense, in that the experimental stimuli are not fully defined by the experimental attributes and may contain additional random variation (Reb \& Cropanzano, 2007) and/or idiosyncratic elements included to enhance the realism of the stimuli (Rosman, Lubatkin, \& O'Neill, 1994). Furthermore, some within-subject experiments do not require participants to rate profiles, but may use a different type of dependent variable. For instance, Rosman et al. (1994) present two types of lenders (commercial vs. venture capital lenders) with hypothetical loan cases and subsequently register the types of information the lenders search for on their computers in response to each loan case. The total amount of financial information and the total amount of strategic information acquired then serve as two dependent variables. As another example, within-subject experiments in which physiological responses to manipulated stimuli are obtained could also be analyzed with the types of models proposed here. For such withinsubject experiments, as for policy capturing data, the earlier mentioned advantages and disadvantages of the SEMWISE approach apply under the same conditions as we discussed there. 
Limitations of the SEMWISE approach include the following. First, even though we did not encounter this in our empirical applications, it is plausible that for complex models fitted to small samples, convergence and (empirical) identification issues might arise. In such instances, if it is not possible to increase the sample size, it might be useful to revert to multilevel regression modeling or to impose some simplifying assumptions (e.g., restraining selected weight factor variances to zero). Future research needs to investigate to what extent such problems occur and how they can be resolved. Second, on a more pragmatic level, data management, data analysis, and interpretation of the results in a SEMWISE framework may be nonstandard and initially challenging, especially for non-SEM users. Whether the additional effort is justified will depend on researchers' familiarity with SEM and the specifics of the research question at hand. At any rate, we hope that the SEMWISE approach opens new opportunities for researchers. 


\section{Appendix A}

\section{Comparison of the SEMWISE and MLM analysis approaches}

This appendix compares the SEMWISE and multi-level modeling (MLM) approaches in terms of data setup, data analysis, and (interpretation of) results as applied to the stylized example shown in Figure 1 of the main text. Figure A1 shows how the data file is structured in the MLM approach (left-hand panel) compared to the SEMWISE approach (right-hand panel), using the four responses of the fictitious respondent in Figure 1 in the main text as an example.

\begin{tabular}{|c|c|c|c|c|}
\hline \multicolumn{5}{|c|}{ Multilevel data setup } \\
\hline Respondent & Profile & $\begin{array}{l}\text { Profile } \\
\text { warmth }\end{array}$ & $\begin{array}{c}\text { Profile } \\
\text { competence }\end{array}$ & Rating \\
\hline 1 & 1 & 1 & -1 & 2 \\
\hline 1 & 2 & -1 & 1 & 4 \\
\hline 1 & 3 & 1 & 1 & 5 \\
\hline 1 & 4 & -1 & -1 & 1 \\
\hline 2 & 1 & $\ldots$ & $\ldots$ & $\ldots$ \\
\hline 2 & 2 & $\ldots$ & $\ldots$ & $\ldots$ \\
\hline$\ldots$ & $\ldots$ & $\ldots$ & $\ldots$ & ... \\
\hline
\end{tabular}

\begin{tabular}{|c|r|r|r|r|}
\hline \multicolumn{6}{|l|}{ SEMWISE data setup } \\
\hline \hline & $\begin{array}{c}\text { Rating for } \\
\text { profile 1 }\end{array}$ & $\begin{array}{c}\text { Rating for } \\
\text { profile 2 }\end{array}$ & $\begin{array}{c}\text { Rating for } \\
\text { profile 3 }\end{array}$ & $\begin{array}{c}\text { Rating for } \\
\text { profile 4 }\end{array}$ \\
\hline Respondent & 2 & 4 & 5 & 1 \\
\hline 1 & $\ldots$ & $\ldots$ & $\ldots$ & $\ldots$ \\
\hline 2 & $\ldots$ & $\ldots$ & $\ldots$ & $\ldots$ \\
\hline 3 &
\end{tabular}

Figure A1. Data setup in a simplified policy capturing study.

As shown in Figure A1, in the MLM dataset, respondents' reactions to the four profiles (obtained by crossing the two levels of warmth with the two levels of competence) are specified on four separate lines of data (i.e., each line represents the rating of one profile, with multiple lines for each respondent). In addition, the profile attribute levels are represented by two additional variables, one containing the effect-coded attribute levels for warmth for each profile, the other containing the effect-coded attribute levels for competence for each profile. The profiles' attribute levels are repeated across respondents, but the ratings are respondent-specific. For the SEMWISE approach, each participant has one line of data and there are four columns of ratings corresponding to the ratings of the four profiles. In other words, MLM requires data in long format, SEMWISE requires data in wide format. In 
the SEMWISE approach, the attribute levels of the profiles are not part of the dataset but are specified as fixed model parameters in the analytical model (see the loadings in Figure 1).

In the MLM approach, the ratings of the teammate profiles are regressed on each profile's warmth and competence level, where (for the sake of this example) effect coding is used to represent the profiles (i.e., -1 represents a low level, +1 represents a high level of an attribute). The estimated regression coefficients are the weights (or utilities) that respondents attach to warmth and competence, which are inferred from the overall ratings of the profiles. In other words, the overall rating of a profile is decomposed into the weights of the attribute levels that characterize the profile. It can be shown that when using MLM, the estimated regression coefficients, which are allowed to vary across respondents, are a compromise between (or weighted combination of) two types of regressions, a pooled regression and individual-level regressions (Gelman \& Hill, 2007). In a pooled regression, it is assumed that all respondents assign the same weights to warmth and competence when rating teammates, so the regression coefficients are fixed to be equal across respondents. In an individual-level regression, it is assumed that each respondent assigns idiosyncratic weights to warmth and competence when rating teammates, so a separate regression equation is estimated for each respondent across the rated profiles (four in the present case). If there is little variability in coefficients across respondents, the MLM estimates will be close to the pooled estimates, but as the variability in coefficients across respondents increases, the MLM estimates move toward the individual-level estimates. The advantage of the MLM regression coefficients is that, on the one hand, the limiting assumption of effect homogeneity is relaxed and that, on the other hand, the estimates tend to be more stable than the individual-level regression coefficients (which are often based on a relatively small number of distinct profiles).

In the SEMWISE approach, the estimated weights are the same as in the MLM approach, but the way in which the model is specified and the weights are estimated is quite different. Instead of regressing teammate ratings on warmth and competence, one specifies a 
factor model in which the ratings of the four experimental profiles are treated as the observed indicators of three latent factors: an intercept factor, a weight factor for warmth, and a weight factor for competence. In order to estimate a factor model, the data have to be structured differently, as shown in Figure A1.

Although the SEMWISE model is a factor model, it should be noted that the SEMWISE factor model differs from a conventional factor model in that the factor loadings are fixed to particular values (analogous to the effect-coded independent variables in the MLM approach) and each indicator (i.e., each profile rating) loads on multiple factors, the reason being that each profile rating is assumed to be a function of the conjoint effect of multiple weight factors (e.g., the rating of the profile with high warmth and high competence depends on the weight that respondents attach to warmth and the weight that respondents attach to competence).

The weight factor scores in the SEMWISE approach are identical to the regression coefficients estimated in the MLM approach. Specifically, the latent intercept factor captures the average rating that each respondent gives to the entire set of profiles. The latent intercept factor has unit loadings on the four indicators (the ratings of the four profiles) and corresponds to the intercept term in the MLM approach. The four profile ratings have loadings on the latent warmth and competence weight factors, and these loadings correspond to the profiles' effect codes (for warmth and competence) in the MLM approach. Thus, both approaches model each profile rating as a function of its effect-coded attribute levels and the respective weights (regression coefficients in MLM, weight factor scores in SEMWISE) of these attribute levels. Since the loadings are effect-coded as -1 for low and +1 for high warmth and competence, respectively, twice the estimated factor score is the difference in the weight attached to low and high warmth or competence.

Below we provide Mplus syntax to simulate data corresponding to the example shown in Figure 1 and to analyze the data in an equivalent way using either SEMWISE or MLM 
(see Figure A1). The Mplus output shows that the parameter estimates are the same across the

MLM and SEMWISE analyses, although the terminology differs across approaches.

1. Data generation

TITLE: Data generation for SEMWISE VS. MLM (Fig 1 and Fig A1); MONTECARLO:

NAMES $=\mathrm{y} 1-\mathrm{y} 4 ;$

NOBS $=250 ;$

SAVE = example_wide.dat;

$\mathrm{SEED}=0$;

MODEL POPULATION:

icept BY y1-y4@1;

wfwarm BY y1@1 y2@-1 y3e1 y4@-1;

!response variables: profile rating;

!sample size

!generated data filename

!results can be replicated exactly

!true model for data generation

! intercept factor

wfcomp BY y1@-1 y2@1 y3@1 y4@-1;

! weight factor for warmth

!weight factor for competence

!factor variances are .7

icept WITH Wfwarm*.1;

icept WITH wfcomp*.1;

wfwarm WITH wfComp*.1;

$\mathrm{y} 1-\mathrm{y}^{4} \cdot 2$;

!factor covariance

!factor covariance

!factor covariance

!residual variance of response

variable

[y1-y4@0] ;

[icept*3 wfwarm*.6 wfcomp*.3];

! intercepts of response variable

! intercept and weight factor means

MODEL: y1-y4 with y1-y4;

!observed sample covariances

2. Data analysis using the SEMWISE approach

TITLE: SEMWISE analysis for the example in Figures 1 and 2;

DATA: FILE = example_wide.dat; !use the data in wide format

VARIABLE: NAMES $=y 1-y^{4}$;

!response variables

MODEL:

icept by y1-y4@1;

wfwarm by y1@1 y2@-1 y3e1 y4@-1;

!intercept factor

wfcomp by y1@-1 y2a1 y3a1 y4a-1;

!weight factor for warmth

[y1-y $4 @ 0]$;

!weight factor for competence

!response intercepts are set to zero

[icept wfwarm wfcomp];

$\mathrm{y} 1-\mathrm{y} 4(\mathrm{e})$;

!freely estimated factor means

!residual variances equal for

correspondence with MLM

OUTPUT: TECH1 STANDARDIZED;

!standardized keyword gives $\mathrm{R}^{2}$

3. Data analysis using the MLM approach

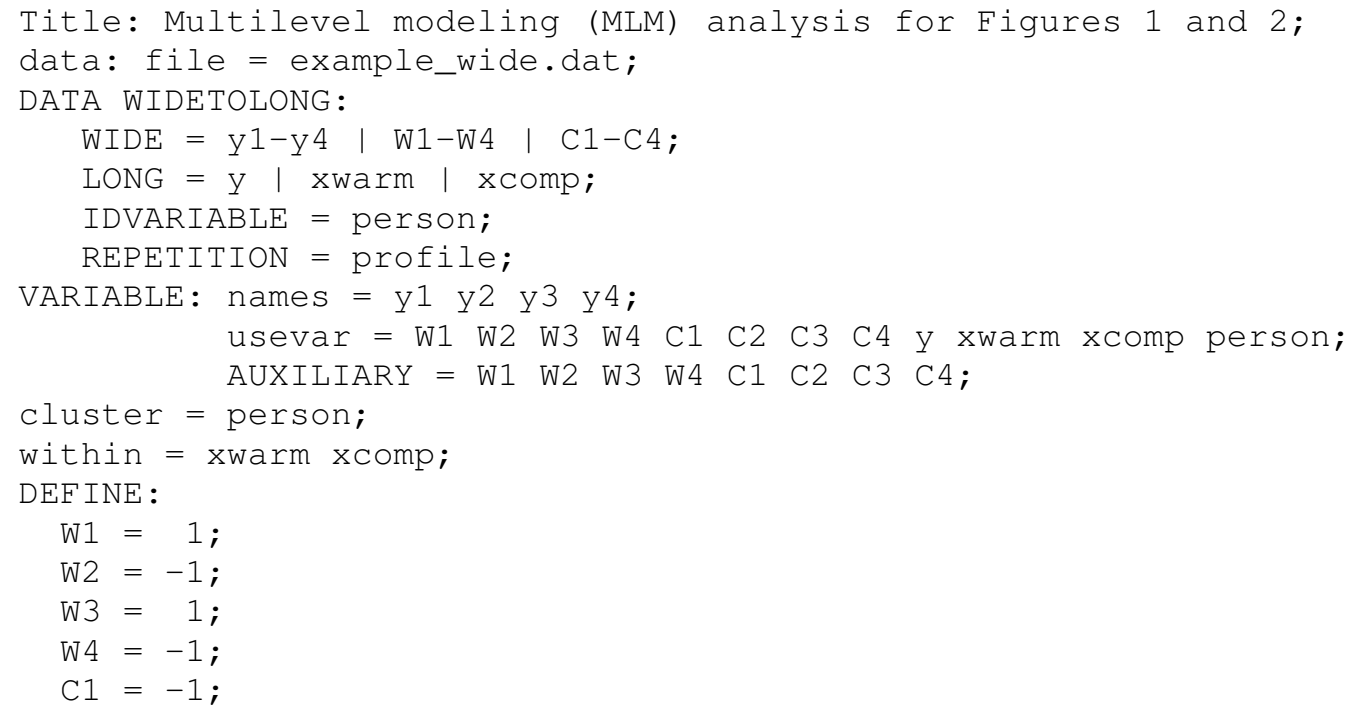




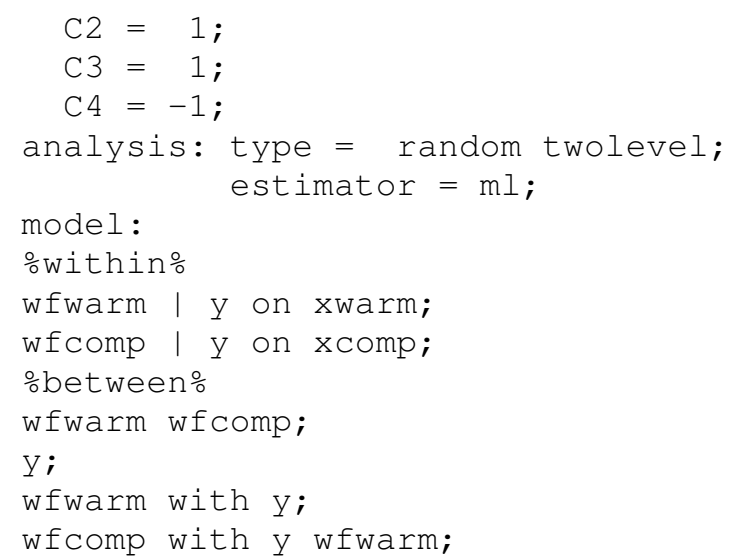

4. Selected output for both approaches

\begin{tabular}{|c|c|c|c|c|}
\hline \multicolumn{5}{|l|}{ SEMWISE } \\
\hline & Est. & S.E. & $t$ & $\mathrm{p}$ \\
\hline WEWARM & WITH & & & \\
\hline ICEPT & 0.138 & 0.049 & 2.815 & 0.005 \\
\hline WECOMP & WITH & & & \\
\hline ICEPT & 0.118 & 0.048 & 2.451 & 0.014 \\
\hline WFWARM & 0.136 & 0.049 & 2.788 & 0.005 \\
\hline Means & & & & \\
\hline ICEPT & 3.036 & 0.055 & 55.193 & 0.000 \\
\hline WFWARM & 0.545 & 0.055 & 9.855 & 0.000 \\
\hline WFCOMP & 0.294 & 0.055 & 5.364 & 0.000 \\
\hline Variano & ces & & & \\
\hline ICEP T & 0.707 & 0.068 & 10.434 & 0.000 \\
\hline WEWARM & 0.715 & 0.069 & 10.442 & 0.000 \\
\hline WFCOMP & 0.700 & 0.067 & 10.426 & 0.000 \\
\hline Residua & al Vari & & & \\
\hline Y1 & 0.196 & 0.018 & 11.180 & 0.000 \\
\hline Y2 & 0.196 & 0.018 & 11.180 & 0.000 \\
\hline Y3 & 0.196 & 0.018 & 11.180 & 0.000 \\
\hline Y4 & 0.196 & 0.018 & 11.180 & 0.000 \\
\hline
\end{tabular}

\begin{tabular}{|c|c|c|c|c|}
\hline \multicolumn{5}{|l|}{ MLM } \\
\hline & Est. & S.E. & t & $\mathrm{p}$ \\
\hline \multicolumn{5}{|c|}{ Between Level } \\
\hline WFWARM & WITH & & & \\
\hline $\mathrm{Y}$ & 0.138 & 0.049 & 2.819 & 0.005 \\
\hline \multicolumn{5}{|c|}{ WFCOMP WITH } \\
\hline Y & 0.118 & 0.046 & 2.565 & 0.010 \\
\hline WEWARM & 0.136 & 0.054 & 2.497 & 0.013 \\
\hline \multicolumn{5}{|l|}{ Means } \\
\hline Y & 3.036 & 0.055 & 55.192 & 0.000 \\
\hline WFWARM & 0.545 & 0.055 & 9.854 & 0.000 \\
\hline WECOMP & 0.294 & 0.055 & 5.363 & 0.000 \\
\hline \multicolumn{5}{|c|}{ Variances } \\
\hline Y & 0.707 & 0.066 & 10.661 & 0.000 \\
\hline WFWARM & 0.715 & 0.065 & 10.992 & 0.000 \\
\hline WFCOMP & 0.700 & 0.069 & 10.185 & 0.000 \\
\hline \multicolumn{5}{|c|}{ Within Level } \\
\hline \multicolumn{5}{|c|}{ Residual Variances } \\
\hline Y & 0.196 & 0.016 & 12.575 & 0.000 \\
\hline
\end{tabular}




\section{Appendix B}

Correlation matrix for empirical application 1

\begin{tabular}{|c|c|c|c|c|c|c|c|c|}
\hline & 1 & 2 & 3 & 4 & 5 & 6 & 7 & 8 \\
\hline 1 Female & & & & & & & & \\
\hline $2 \mathrm{HHL}$ & .009 & & & & & & & \\
\hline $3 \mathrm{HLL}$ & .039 & .481 & & & & & & \\
\hline $4 \mathrm{HHH}$ & -.023 & 420 & .297 & & & & & \\
\hline $5 \mathrm{HLH}$ & -.062 & .222 & .376 & .418 & & & & \\
\hline $6 \mathrm{LHL}$ & .055 & .547 & .382 & .358 & .103 & & & \\
\hline 7 LLL & .027 & 432 & .585 & .280 & .344 & .415 & & \\
\hline $8 \mathrm{LHH}$ & .043 & .415 & .337 & .556 & .337 & .418 & .289 & \\
\hline $9 \mathrm{LLH}$ & .045 & .169 & .398 & .362 & .595 & .188 & .459 & .414 \\
\hline
\end{tabular}

Note: Correlations based on data setup in wide format (responses to the experimental profiles correspond to variables 2 to 9). Female refers to respondent gender $(0=$ male, $1=$ female $)$. The other variable names refer to the levels $(\mathrm{L}=$ low, $\mathrm{H}=$ high $)$ of the three attributes attractiveness, warmth and competence. 
Appendix C

Correlation matrix of empirical application 2

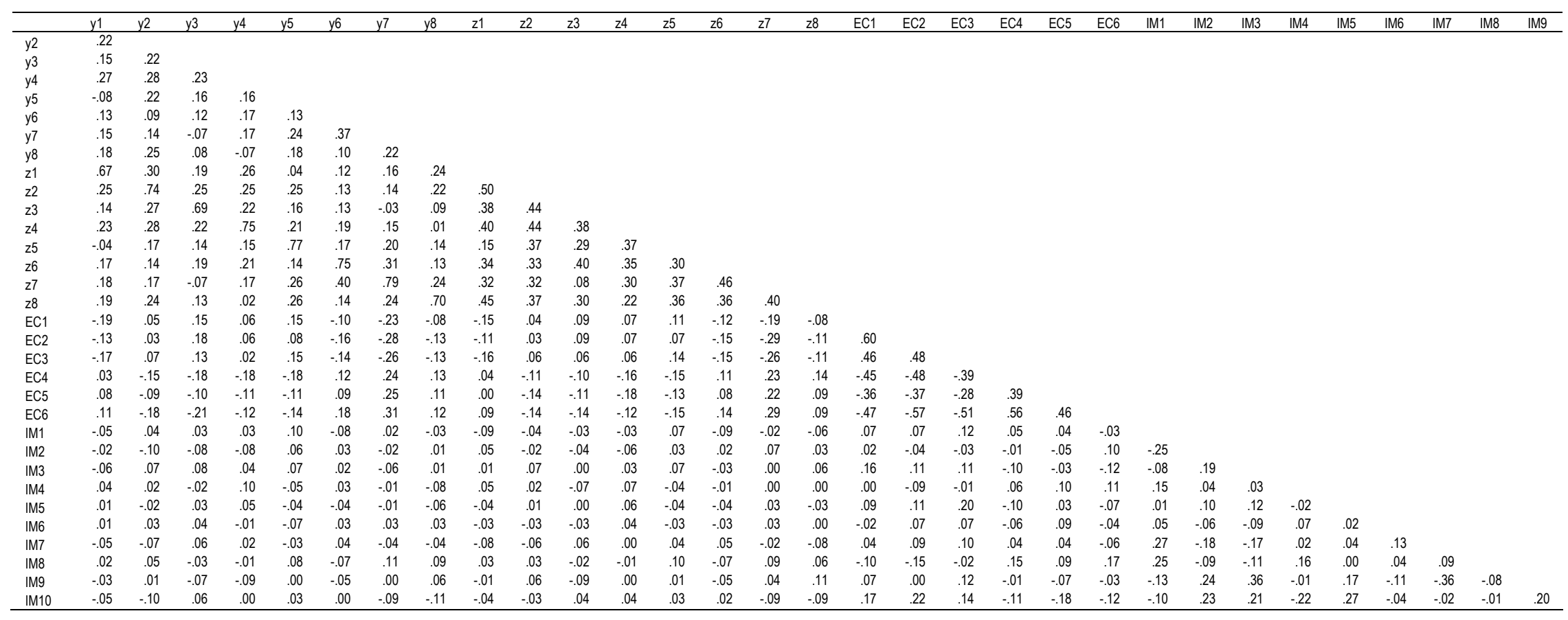

Note: Correlations based on data setup in wide format. Variables y1-y8 refer to profile ratings using the first item ('To what extent would you like to work for this company?' rated by manipulating a sliding bar linked to a smiley face with five levels ranging from 1= low to 5=high), z1-z8 to profile ratings using the second item ('How attractive do you find this company?' rated by means of a continuous sliding scale marked by five stars with scores ranging from zero to five). EC1-EC6 refer to the Environmental Concern scale items, IM1-IM10 to the Impression Management scale items. 


\section{REFERENCES}

Aguinis, H., \& Bradley, K. J. (2014). Best practice recommendations for designing and implementing experimental vignette methodology studies. Organizational Research Methods, 17(4), 351-371. doi: 10.1177/1094428114547952

Aiman-Smith, L., Bauer, T. N., \& Cable, D. M. (2001). Are you attracted? Do you intend to pursue? A recruiting policy-capturing study. Journal of Business and Psychology, 16(2), 219-237. doi: 10.1023/A:1011157116322

Aiman-Smith, L., Scullen, S. E., \& Barr, S. H. (2002). Conducting studies of decision making in organizational contexts: A tutorial for policy-capturing and other regression-based techniques. Organizational Research Methods, 5(4), 388-414. doi: 10.1177/109442802237117

Bollen, K.A. (1989). Structural equations with latent variables, Wiley, New York.Caruso, E. M., Rahnev, D. A., \& Banaji, M. R. (2009). Using conjoint analysis to detect discrimination: revealing covert preferences from overt choices. Social Cognition, 27(1), 128-137. doi: 10.1521/soco.2009.27.1.128

Catlin, J. R., \& Wang, Y. (2012). Recycling gone bad: When the option to recycle increases resource consumption. Journal of Consumer Psychology. doi: 10.1016/j.jcps.2012.04.001

Cheung, G. W., \& Rensvold, R. B. (2001). The effects of model parsimony and sampling error on the fit of structural equation models. Organizational Research Methods, 4(3), 236-264. doi: 10.1177/109442810143004

Connelly, B. L., Ketchen, D. J., Gangloff, K. A., \& Shook, C. L. (2016). Investor perceptions of CEO successor selection in the wake of integrity and competence failures: A policy capturing study. Strategic management journal, 37(10), 2135-2151. doi: $10.1002 /$ smj. 2430

Cuddy, A. J., Glick, P., \& Beninger, A. (2011). The dynamics of warmth and competence judgments, and their outcomes in organizations. Research in Organizational Behavior, 31, 73-98. doi: 10.1016/j.riob.2011.10.004

Curran, P. J. (2003). Have multilevel models been structural equation models all along? Multivariate Behavioral Research, 38(4), 529-568. doi: 10.1207/s15327906mbr3804_5

Edwards, J. R. (2001). Multidimensional constructs in organizational behavior research: An integrative analytical framework. Organizational Research Methods, 4(2), 144-192. doi: Doi 10.1177/109442810142004

Feingold, A. (2009). Effect sizes for growth-modeling analysis for controlled clinical trials in the same metric as for classical analysis. Psychological Methods, 14(1), 45-53. doi: $10.1037 / \mathrm{a} 0014699$

Fink, B., \& Penton-Voak, I. (2002). Evolutionary psychology of facial attractiveness. Current Directions in Psychological Science, 11(5), 154-158. doi: 10.1111/1467-8721.00190

Fiske, S. T., Cuddy, A. J., Glick, P., \& Xu, J. (2002). A model of (often mixed) stereotype content: competence and warmth respectively follow from perceived status and competition. Journal of Personality and Social Psychology, 82(6), 878-902. doi: 10.1037//0022-3514.82.6.878

Gelman, A., \& Hill, J. (2007). Data analysis using regression and multilevelhierarchical models (Vol. 1): Cambridge University Press New York, NY, USA.

Graham, M. E., \& Cable, D. M. (2001). Consideration of the incomplete block design for policy-capturing research. Organizational Research Methods, 4(1), 26-45. doi: $10.1177 / 109442810141002$

Grégoire, D. A., \& Shepherd, D. A. (2012). Technology-market combinations and the identification of entrepreneurial opportunities: An investigation of the opportunity- 
individual nexus. Academy of Management Journal, 55(4), 753-785. doi: 10.5465/amj.2011.0126

Helm, J. L., Castro-Schilo, L., \& Oravecz, Z. (2017). Bayesian Versus Maximum Likelihood Estimation of Multitrait-Multimethod Confirmatory Factor Models. Structural Equation Modeling: A Multidisciplinary Journal, 24(1), 17-30. Doi: 10.1080/10705511.2016.1236261

Hinkin, T. R. (1998). A brief tutorial on the development of measures for use in survey questionnaires. Organizational Research Methods, 1(1), 104-121. doi: $10.1177 / 109442819800100106$

Jones, D. A., \& Willness, C. R. (2013). Corporate social performance, organizational reputation, and recruitment. In D. M. Cable \& K. Y. T. Yu (Eds.), The Oxford handbook of recruitment. Oxford: Oxford University Press.

Karren, R. J., \& Barringer, M. W. (2002). A review and analysis of the policy-capturing methodology in organizational research: Guidelines for research and practice. Organizational Research Methods, 5(4), 337-361. doi: 10.1177/109442802237115

Kline, R. B. (2016), Principles and practice of structural equation modeling, $4^{\text {th }}$ ed., New York: The Guilford Press.

Kristof-Brown, A. L., Jansen, K. J., \& Colbert, A. E. (2002). A policy-capturing study of the simultaneous effects of fit with jobs, groups, and organizations. Journal of Applied Psychology, 87(5), 985. doi: 10.1037//0021-9010.87.5.985

LaHuis, D. M., Hartman, M. J., Hakoyama, S., \& Clark, P. C. (2014). Explained variance measures for multilevel models. Organizational Research Methods, 17(4), 433-451. doi: $10.1177 / 1094428114541701$

Lance, C. E., Butts, M. M., \& Michels, L. C. (2006). The sources of four commonly reported cutoff criteria - What did they really say? Organizational Research Methods, 9(2), 202-220. doi: 10.1177/1094428105284919

Lance, C. E., Noble, C. L., \& Scullen, S. E. (2002). A critique of the correlated traitcorrelated method and correlated uniqueness models for multitrait-multimethod data. Psychological Methods, 7(2), 228. doi: 10.1037/1082-989X.7.2.228

Ma, D. S., Correll, J., \& Wittenbrink, B. (2015). The Chicago face database: A free stimulus set of faces and norming data. Behavior research methods, 47(4), 1122-1135. doi: $10.3758 / \mathrm{s} 13428-014-0532-5$

Maydeu-Olivares, A., \& Coffman, D. L. (2006). Random intercept item factor analysis. Psychological Methods, 11(4), 344-362. doi: 10.1037/1082-989X.11.4.344

Mulaik, S. A. (2009). Linear causal modeling with structural equations: CRC Press.

Muthén, L. K., \& Muthén, B. O. (2002). How to use a Monte Carlo study to decide on sample size and determine power. Structural Equation Modeling, 9(4), 599-620. doi: 10.1207/S15328007SEM0904_8

Newman, D. A., \& Lyon, J. S. (2009). Recruitment Efforts to Reduce Adverse Impact: Targeted Recruiting for Personality, Cognitive Ability, and Diversity. Journal of Applied Psychology, 94(2), 298-317. doi: 10.1037/a0013472

Olsen, J. E., \& Martins, L. L. (2016). Racioethnicity, community makeup, and potential employees' reactions to organizational diversity management approaches. Journal of Applied Psychology, 101(5), 657. doi: 10.1037/ap10000080

Patzelt, H., \& Shepherd, D. A. (2008). The decision to persist with underperforming alliances: The role of trust and control. Journal of Management Studies, 45(7), 12171243. doi: DOI 10.1111/j.1467-6486.2008.00791.x

Paulhus, D. L. (1991). Measurement and control of response bias. In J. P. Robinson, P. R. Shaver \& L. S. Wrightsman (Eds.), Measures of Personality and Social Psychological Attitudes: Academic Press. 
Ployhart, R. E., \& Oswald, F. L. (2004). Applications of mean and covariance structure analysis: Integrating correlational and experimental approaches. Organizational Research Methods, 7(1), 27-65.

Reb, J., \& Cropanzano, R. (2007). Evaluating dynamic performance: The influence of salient gestalt characteristics on performance ratings. Journal of Applied Psychology, 92(2), 490-499. DOI: 10.1037/0021-9010.92.2.490

Rhemtulla, M. (2016). Population Performance of SEM Parceling Strategies Under Measurement and Structural Model Misspecification. Psychological Methods, 21(3), 348-368. doi: 10.1037/met0000072

Rosman, A., Lubatkin, M., \& O'Neill, H. (1994). Rigidity in decision behaviors: A withinsubject test of information acquisition using strategic and financial informational cues. Academy of Management Journal, 37(4), 1017-1033. DOI: 10.2307/256610

Rotundo, M., \& Sackett, P. R. (2002). The relative importance of task, citizenship, and counterproductive performance to global ratings of job performance: a policycapturing approach. Journal of Applied Psychology, 87(1), 66-80. doi: 10.1037/00219010.87.1.66

Sauer, C., Auspurg, K., Hinz, T., \& Liebig, S. (2011). The application of factorial surveys in general population samples: The effects of respondent age and education on response times and response consistency. Survey Research Methods, 5(3), 89-102. doi: 10.18148/srm/2011.v5i3.4625\#sthash.YYreWVGE.dpuf

Shepherd, D. A., Patzelt, H., \& Baron, R. A. (2013). "I Care about Nature, but ...": Disengaging Values in Assessing Opportunities that Cause Harm. Academy of Management Journal, 56(5), 1251-1273. doi: 10.5465/amj.2011.0776

Tomassetti, A. J., Dalal, R. S., \& Kaplan, S. A. (2016). Is Policy Capturing Really More Resistant Than Traditional Self-Report Techniques to Socially Desirable Responding? Organizational Research Methods, 19(2), 255-285. doi: 10.1177/1094428115627497

Vandenberg, R. J., \& Lance, C. E. (2000). A review and synthesis of the measurement invariance literature: Suggestions, practices, and recommendations for organizational research. Organizational Research Methods, 3(1), 4-70. doi: $10.1177 / 109442810031002$

Weijters, B., Baumgartner, H., \& Schillewaert, N. (2013). Reversed Item Bias: An Integrative Model. Psychological Methods, 18(3), 320-334. doi: 10.1037/a0032121 
Table 1

Some examples of policy capturing applications

\begin{tabular}{|c|c|c|}
\hline $\begin{array}{l}\text { Dependent variable and type of } \\
\text { experimental stimuli }\end{array}$ & Manipulated attributes & Source \\
\hline $\begin{array}{l}\text { Organizational attractiveness based on } \\
\text { job and organization descriptions }\end{array}$ & $\begin{array}{l}\text { Pay } \\
\text { Promotion } \\
\text { Layoff policy } \\
\text { Ecological rating }\end{array}$ & $\begin{array}{l}\text { Aiman-Smith et al. } \\
\text { (2001) }\end{array}$ \\
\hline $\begin{array}{l}\text { Choice of prospective teammates for a } \\
\text { trivia contest }\end{array}$ & $\begin{array}{l}\text { Education } \\
\text { IQ } \\
\text { Experience } \\
\text { Body weight }\end{array}$ & Caruso et al. (2009) \\
\hline $\begin{array}{l}\text { Evaluation of potential CEO successors } \\
\text { after failure }\end{array}$ & $\begin{array}{l}\text { Type of failure: competence vs. integrity } \\
\text { Outsider, interim, insider }\end{array}$ & $\begin{array}{l}\text { Connelly, Ketchen, } \\
\text { Gangloff, and Shook } \\
\text { (2016) }\end{array}$ \\
\hline $\begin{array}{l}\text { Fit and feasibility of entrepreneurial } \\
\text { opportunities (technology-market } \\
\text { combinations) }\end{array}$ & $\begin{array}{l}\text { Superficial market-technology similarities } \\
\text { Structural market-technology similarities }\end{array}$ & $\begin{array}{l}\text { Grégoire and Shepherd } \\
\text { (2012) }\end{array}$ \\
\hline Work satisfaction as a function of fit & $\begin{array}{l}\text { Person-job fit } \\
\text { Person-group fit } \\
\text { Person-organization fit }\end{array}$ & $\begin{array}{l}\text { Kristof-Brown, Jansen, } \\
\text { and Colbert (2002) }\end{array}$ \\
\hline $\begin{array}{l}\text { Organizational attractiveness based on } \\
\text { features of organizational image }\end{array}$ & $\begin{array}{l}\text { Type of company (demanding, results-oriented, detail- } \\
\text { oriented, and innovative) } \\
\text { Type of applicant sought (self-disciplined, conscientious, } \\
\text { reliable, well-organized, hard-working, sharp, intelligent, } \\
\text { brilliant, logical, and smart) }\end{array}$ & $\begin{array}{l}\text { Newman and Lyon } \\
\text { (2009) }\end{array}$ \\
\hline $\begin{array}{l}\text { Organizational attractiveness based on } \\
\text { signals about organizations' } \\
\text { diversity management (DM) approach }\end{array}$ & $\begin{array}{l}\text { Value type (none specified, terminal, instrumental, dual) } \\
\text { Acculturation strategy (neutral, assimilation, integration) }\end{array}$ & $\begin{array}{l}\text { Olsen and Martins } \\
\text { (2016) }\end{array}$ \\
\hline
\end{tabular}




\begin{tabular}{|c|c|c|}
\hline Decision to persist with strategic alliances & $\begin{array}{l}\text { Output } \\
\text { Behavioral control } \\
\text { Social control } \\
\text { Competence trust } \\
\text { Goodwill trust }\end{array}$ & $\begin{array}{l}\text { Patzelt and Shepherd } \\
(2008)\end{array}$ \\
\hline $\begin{array}{l}\text { Evaluations of dynamic performance of } \\
\text { sales people }\end{array}$ & $\begin{array}{l}\text { Performance trend } \\
\text { Performance mean } \\
\text { Performance variation }\end{array}$ & $\begin{array}{l}\text { Reb and Cropanzano } \\
(2007)^{a}\end{array}$ \\
\hline $\begin{array}{l}\text { Strategic and financial information } \\
\text { acquisition in assessing loan cases }\end{array}$ & Structured vs. ill-structured settings & Rosman et al. (1994) ${ }^{b}$ \\
\hline $\begin{array}{l}\text { Global ratings of job performance based } \\
\text { on aspects of performance }\end{array}$ & $\begin{array}{l}\text { Employees' task performance } \\
\text { Citizenship performance } \\
\text { Counterproductive performance }\end{array}$ & $\begin{array}{l}\text { Rotundo and Sackett } \\
\text { (2002) }\end{array}$ \\
\hline $\begin{array}{l}\text { Assessment of attractiveness of } \\
\text { entrepreneurial opportunities }\end{array}$ & $\begin{array}{l}\text { Value } \\
\text { Rarity } \\
\text { Inimitability } \\
\text { Impact on the natural environment }\end{array}$ & Shepherd et al. (2013) \\
\hline $\begin{array}{l}\text { Organizational attractiveness based on job } \\
\text { and organization descriptions }\end{array}$ & $\begin{array}{l}\text { Organization offering essential services/products to the public } \\
\text { Opportunity to use skills and abilities } \\
\text { Autonomy and independence } \\
\text { Responsibility and leadership opportunities } \\
\text { Pay and fringe benefits } \\
\text { Flexibility in scheduling work hours and vacations }\end{array}$ & $\begin{array}{l}\text { Tomassetti et al. } \\
\text { (2016) }\end{array}$ \\
\hline
\end{tabular}

$\mathrm{a}, \mathrm{b}=$ These studies are not policy capturing studies in the narrow sense: The profiles were not fully defined by the manipulated attributes since there were additional differences between the profiles. In ${ }^{\text {a }}$, performance curves were generated based on the trend, mean and standard deviation specification, with addition of random error. In ${ }^{\mathrm{b}}$, each of four loan cases contained idiosyncratic elements to enhance external validity. 
Table 2

Parameters as a function of number of attributes

\begin{tabular}{cccc}
\hline $\begin{array}{c}\text { Factorial design (number } \\
\text { of observed variables, } \mathrm{p})\end{array}$ & $\begin{array}{c}\text { Number of unique observed } \\
\text { variances/covariances and means }\end{array}$ & $\begin{array}{c}\text { Parameters estimated in a main- } \\
\text { effects model (assuming distinct } \\
\text { error variances) }\end{array}$ & $\begin{array}{c}\text { Parameters estimated in a } \\
\text { saturated model }\end{array}$ \\
\hline $2^{2}$ & 14 & 22 & 13 \\
$2^{3}$ & 44 & 36 & 152 \\
$2^{4}$ & 152 & $\frac{k(k+3)}{2}+p$ & $\frac{p(p+3)}{2}$ \\
\hline
\end{tabular}

Note: $\mathrm{k}$ is the number of dichotomous experimental factors; $\mathrm{p}$ is the number of observed variables (responses). In the saturated model, all possible interactions are included, but the error variances are restricted to zero. 
Table 3

Stimulus construction for empirical application 1

\begin{tabular}{|c|c|c|c|c|}
\hline Attribute & Element & Source/reference & Levels & Set \\
\hline Attractiveness & [Picture] & $\begin{array}{l}\text { Chicago Face Database (Ma, } \\
\text { Correll, \& Wittenbrink, 2015), } \\
\text { adapted to a } 160 \text { × } 112 \text { format }\end{array}$ & $\begin{array}{l}\text { below } \\
\text { average } \\
\text { above } \\
\text { average }\end{array}$ & $\begin{array}{l}12 \text { pictures of white women close to attractiveness score of } \\
2.57 \text { (i.e., } M-1 \mathrm{SD} \text { ) } \\
12 \text { pictures of white women close to attractiveness score of } \\
4.34 \text { (i.e., } \mathrm{M}+1 \mathrm{SD} \text { ) }\end{array}$ \\
\hline I & [Name] & listofrandomnames.com & N.A. & $\begin{array}{l}\text { Jackelyn, Alison, Gabriele, Lorretta, Dottie, Marybeth, } \\
\text { Sarina, Hester, Janet, Rosemary }\end{array}$ \\
\hline Warmth & [warmth] & $\begin{array}{l}\text { Fiske, Cuddy, Glick, and Xu } \\
\text { (2002) }\end{array}$ & $\begin{array}{l}\text { high } \\
\text { low }\end{array}$ & $\begin{array}{l}\text { friendly, trustworthy, warm, good-natured } \\
\text { not always kind, occasionally unfriendly, sometimes aloof, } \\
\text { a bit standoffish }\end{array}$ \\
\hline Competence & [IQ] & Caruso et al. (2009) & $\begin{array}{l}\text { average } \\
\text { high }\end{array}$ & $\begin{array}{l}\text { randomly sampled number from the range [100-104] } \\
\text { (inclusive) } \\
\text { randomly sampled number from the range [118-122] } \\
\text { (inclusive) }\end{array}$ \\
\hline
\end{tabular}


Table 4

Parameter estimates for the one-group analysis in empirical application 1

\begin{tabular}{llcccc}
\hline Parameter & Weight factor & Est. & SE & $\mathrm{t}$ & $\mathrm{p}$ \\
\hline Means & Intercept & 2.83 & .04 & 72.30 & $<.001$ \\
& Competent & .64 & .02 & 28.95 & $<.001$ \\
& Warm & .50 & .02 & 23.09 & $<.001$ \\
& Attractive & .06 & .02 & 3.74 & $<.001$ \\
& & & & & \\
\hline Variances & Intercept & .35 & .04 & 10.09 & $<.001$ \\
& Competent & .08 & .01 & 7.18 & $<.001$ \\
& Warm & .08 & .01 & 7.03 & $<.001$ \\
& Attractive & .02 & .01 & 2.64 & .008 \\
& & & & & \\
\hline
\end{tabular}


Table 5

Parameter estimates for the two-group model in empirical illustration 1

\begin{tabular}{|c|c|c|c|c|c|c|c|c|c|c|c|c|c|}
\hline & & \multicolumn{4}{|l|}{ M } & \multicolumn{4}{|l|}{ F } & \multicolumn{4}{|l|}{ Diff. } \\
\hline & & Est. & SE & $\mathrm{t}$ & $\mathrm{p}$ & Est. & SE & $\mathrm{t}$ & $\mathrm{p}$ & Est. & SE & $\mathrm{t}$ & $\mathrm{p}$ \\
\hline \multirow[t]{4}{*}{ Mean } & Intercept & 2.820 & .052 & 54.696 & $<.001$ & 2.849 & .060 & 47.593 & $<.001$ & -.029 & .079 & -.372 & .710 \\
\hline & Attractive & .079 & .023 & 3.424 & .001 & .034 & .019 & 1.753 & .080 & .045 & .030 & 1.494 & .135 \\
\hline & Warm & .490 & .030 & 16.413 & $<.001$ & .504 & .031 & 16.145 & $<.001$ & -.014 & .043 & -.330 & .741 \\
\hline & Competent & .657 & .029 & 22.757 & $<.001$ & .619 & .034 & 18.171 & $<.001$ & .038 & .045 & .845 & .398 \\
\hline \multirow{4}{*}{ Variance } & Intercept & .318 & .044 & 7.227 & $<.001$ & .394 & .056 & 7.035 & $<.001$ & -.076 & .071 & -1.064 & .288 \\
\hline & Attractive & .025 & .009 & 2.756 & .006 & .002 & .006 & .345 & .730 & .023 & .011 & 2.051 & .040 \\
\hline & Warm & .075 & .015 & 5.035 & $<.001$ & .076 & .015 & 4.883 & $<.001$ & -.001 & .021 & -.026 & .979 \\
\hline & Competent & .066 & .014 & 4.754 & $<.001$ & .098 & .018 & 5.342 & $<.001$ & -.032 & .023 & -1.378 & .168 \\
\hline
\end{tabular}

Note: $\mathrm{M}=$ men, $\mathrm{F}=$ women, Diff. = difference between the parameter estimates for men vs. women. 
Table 6

Attributes, profiles (stimulus coding) and estimation results, empirical application 2

\begin{tabular}{|c|c|c|c|c|c|c|c|c|c|c|c|c|c|c|c|c|c|}
\hline \multirow[t]{2}{*}{ Attribute } & \multicolumn{2}{|c|}{ Levels } & \multicolumn{8}{|c|}{ Stimuli specification / Loadings } & \multicolumn{2}{|l|}{$\mathrm{M}$} & \multicolumn{2}{|l|}{ Var. } & \multicolumn{3}{|c|}{ Correlations } \\
\hline & -1 & 1 & 1 & 2 & 3 & 4 & 5 & 6 & 7 & 8 & Est. & SE & Est. & SE & 1 & 2 & 3 \\
\hline $\begin{array}{l}\text { 1. COMP: The firm's } \\
\text { compensation package is } \\
\text { somewhat }[x] \text { average for } \\
\text { the industry }\end{array}$ & below & above & -1 & -1 & -1 & 1 & 1 & 1 & 1 & -1 & .48 & .02 & .04 & .01 & & & \\
\hline $\begin{array}{l}\text { 2. PROMO: the typical } \\
\text { career path for the average } \\
\text { graduate includes }[\mathrm{x}] \\
\text { promotion in five years }\end{array}$ & no & one & -1 & 1 & -1 & -1 & 1 & -1 & 1 & 1 & .46 & .02 & .04 & .01 & .28 & & \\
\hline $\begin{array}{l}\text { 3. LAYOFF: The } \\
\text { company's policy is that } \\
\text { employees are [x] laid off. }\end{array}$ & sometimes & rarely & 1 & 1 & -1 & 1 & -1 & -1 & 1 & -1 & .28 & .02 & .03 & .01 & -.01 & -.40 & \\
\hline $\begin{array}{l}\text { 4. CEC: Concern for the } \\
\text { environment is }[\mathrm{x}] \text { priority } \\
\text { in the company }\end{array}$ & not a & $\mathrm{a}$ & -1 & 1 & 1 & 1 & 1 & -1 & -1 & -1 & .26 & .02 & .05 & .01 & -.10 & -.32 & -.15 \\
\hline
\end{tabular}

Note: The response variable was scaled from zero to ten. The intercept factor has unit loadings, but was not part of the cards (stimuli) presented to respondents. Correlations with $\mathrm{p}<.05$ are printed in boldface. All mean and variance estimates are significantly different from zero $(\mathrm{p}<.05) . \mathrm{COMP}=$ Compensation; $\mathrm{PROMO}=$ Promotion opportunities; LAYOFF $=$ Layoff policy; CEC $=$ Company's environmental concern. 
Table 7

Comparison of the Multilevel (Mixed) Model and the SEMWISE Approach Multilevel (Mixed) Model

SEMWISE Approach

Primary purpose of To study the influence of categorical or continuous the analysis independent variables (IVs) on a dependent variable (DV) in situations in which the data have a

Same as in the multilevel model. Additionally, both antecedents and outcomes can be related to the weight hierarchical structure. In a within-subject design, the factors (i.e., the individually varying effects of IVs on repeated observations are nested within respondent and the IVs are categorical. Determinants of the variation in the level-1 random coefficients can be studied in the level-2 model. DVs).

Measurement No explicit measurement model for the DV or the
determinants of the random coefficients in the level-2 model. If the IVs in the level-1 model are

model

manipulations, no measurement model is needed.

It is possible to specify a factor model for the DVs if

multiple indicators of each DV are available.

Alternatively, unreliability of measurement can be accounted for by incorporating an external measure of reliability (e.g., coefficient alpha) into the analysis. Unreliability of measurement in the determinants and consequents of the random coefficient factors can also be modeled. Measurement quality can be assessed based on the usual statistics (e.g., composite reliability).

Assumptions about Many different error structures are possible. Model the error structure comparisons can be conducted to find the most of the repeated appropriate error structure, in an effort to strike a measures balance between type I and type II errors.

Fit assessment Models can be compared based on information theory measures (AIC, BIC, etc.). A likelihood ratio test of whether the variances and covariances of the random effects are nonzero is also available.

Same as in the mixed model, although the different error structures have not been preprogrammed in existing software and have to be implemented by the researcher.

All fit assessments available in mixed models can be used. Additionally, all fit indices used in structural equation modeling are available (chi-square goodness of fit test, absolute and incremental fit indices).

Variance explained Alternative measures of variance explained are available, even though there does not seem to be a consensus on which ones to use and report; but see LaHuis, Hartman, Hakoyama, and Clark (2014)
The variance explained in each observed variable (repeated measure) is readily available. This indicates how well the experimental factors account for the variation in a profile's ratings across respondents. 


\section{Questionnaire}

SEMWISE model

Profiles

Profile ratings

Attribute levels as loadings

Weight factors

1

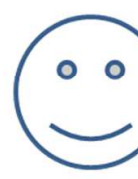

2
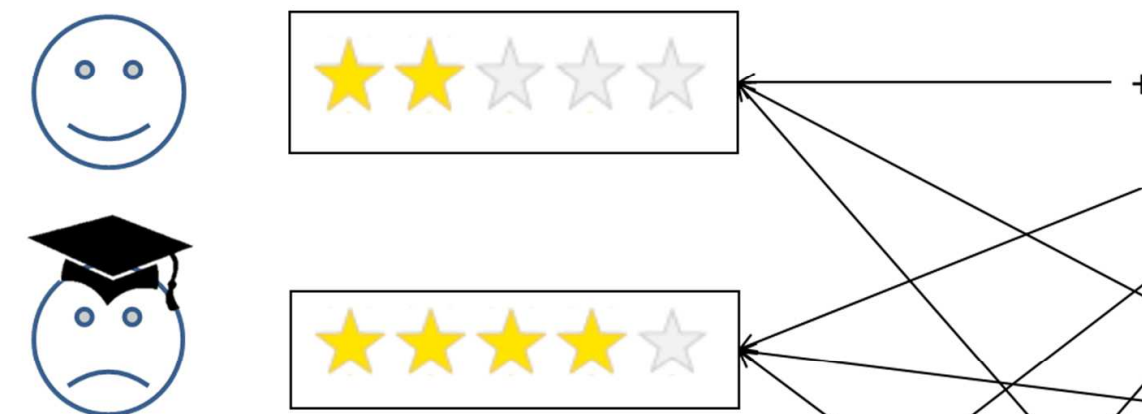

$+1$

$-1$

$+1-1$
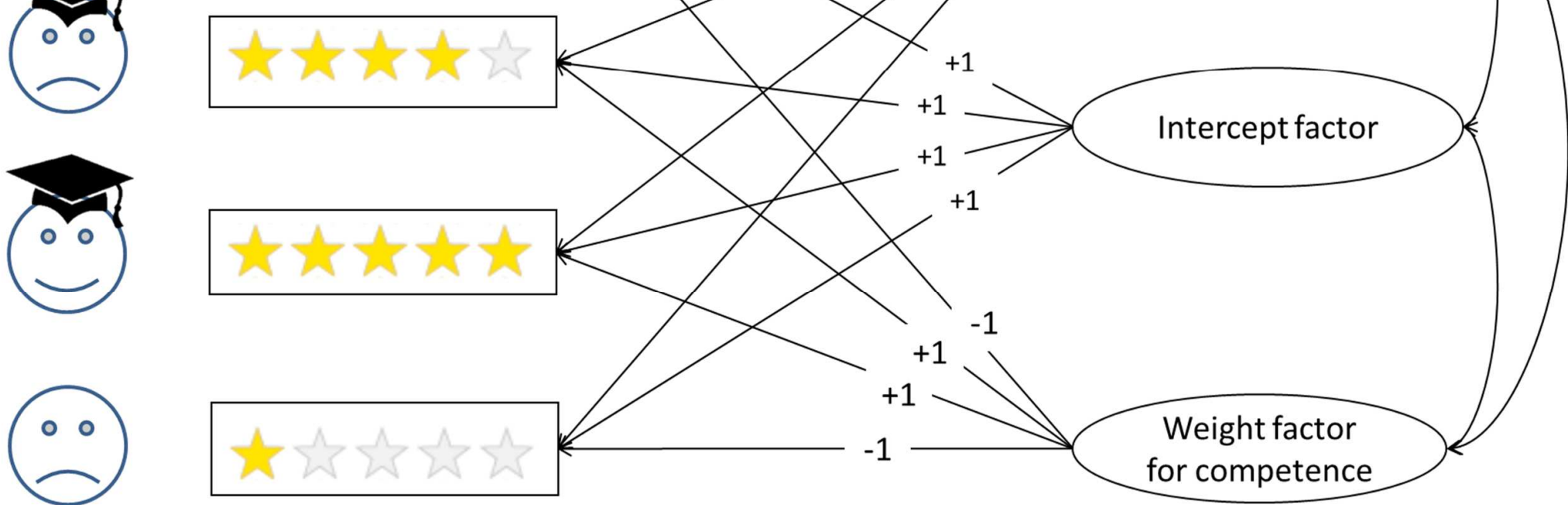

4

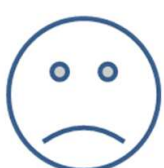

Figure 1. Stylized policy capturing example using a SEMWISE model. Respondents rate four experimental teammate profiles varying in warmth (high $=$ smiling face vs. low = sad face) and competence (high = graduation cap vs. low = no cap) on a 5-star scale (left). In SEMWISE, these ratings are used as indicators of two weight factors plus an intercept factor (right). Since the profiles consist of combinations of warmth and competence, the profile ratings are modeled as combinations of warmth and competence weights. Respondents who value warmth (competence) will rate the first and third (second and third) profiles higher relative to the second and fourth (first and fourth) profiles, controlling for competence (warmth). The intercept factor has unit loadings for all four profiles and thus captures individuals' average ratings of the four profiles, regardless of competence and warmth. 


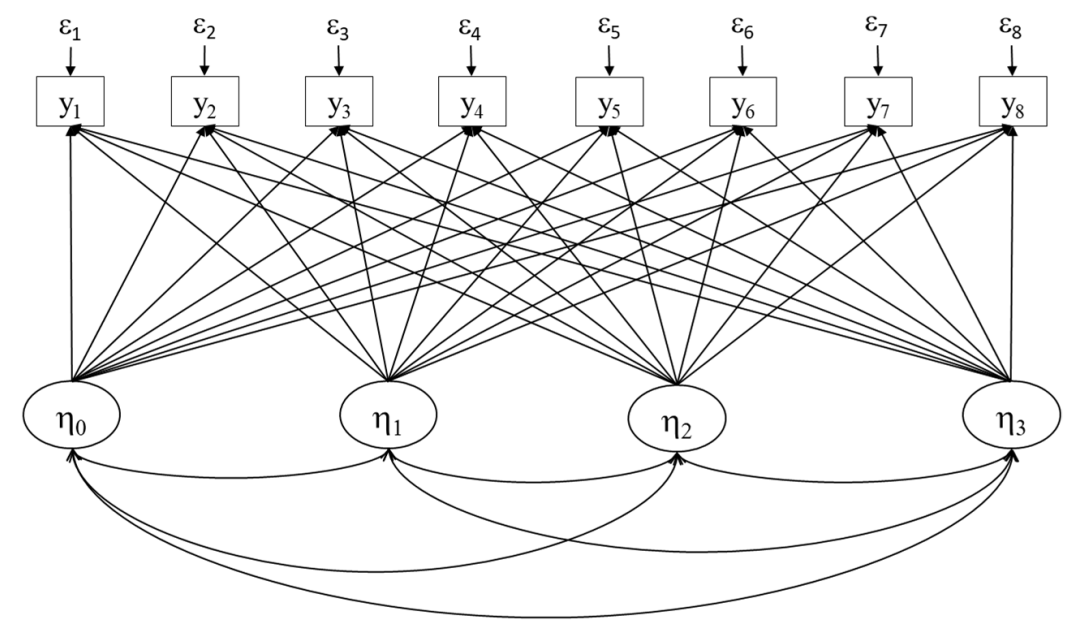

$$
\left[\begin{array}{l}
y_{1} \\
y_{2} \\
y_{3} \\
y_{4} \\
y_{5} \\
y_{6} \\
y_{7} \\
y_{8}
\end{array}\right]=\left[\begin{array}{llll}
1 & +1 & +1 & +1 \\
1 & -1 & +1 & +1 \\
1 & +1 & -1 & +1 \\
1 & -1 & -1 & +1 \\
1 & +1 & +1 & -1 \\
1 & -1 & +1 & -1 \\
1 & +1 & -1 & -1 \\
1 & -1 & -1 & -1
\end{array}\right] \cdot\left[\begin{array}{l}
\eta_{0} \\
\eta_{1} \\
\eta_{2} \\
\eta_{3}
\end{array}\right]+\left[\begin{array}{l}
\varepsilon_{1} \\
\varepsilon_{2} \\
\varepsilon_{3} \\
\varepsilon_{4} \\
\varepsilon_{5} \\
\varepsilon_{6} \\
\varepsilon_{7} \\
\varepsilon_{8}
\end{array}\right]
$$

Figure 2. SEMWISE model for three binary attributes. The indicators $\mathrm{y}_{1}-\mathrm{y}_{8}$ represent individuals' ratings of the eight stimuli and load on four latent factors; the first factor $\left(\eta_{0}\right)$ captures the mean rating across all stimuli, the other three $\left(\eta_{1}, \eta_{2}, \eta_{3}\right)$ capture the effects of each of the three attributes. For readability, Figure 2 does not include the mean structure, which includes four factor means (the item intercepts are restricted to zero). 


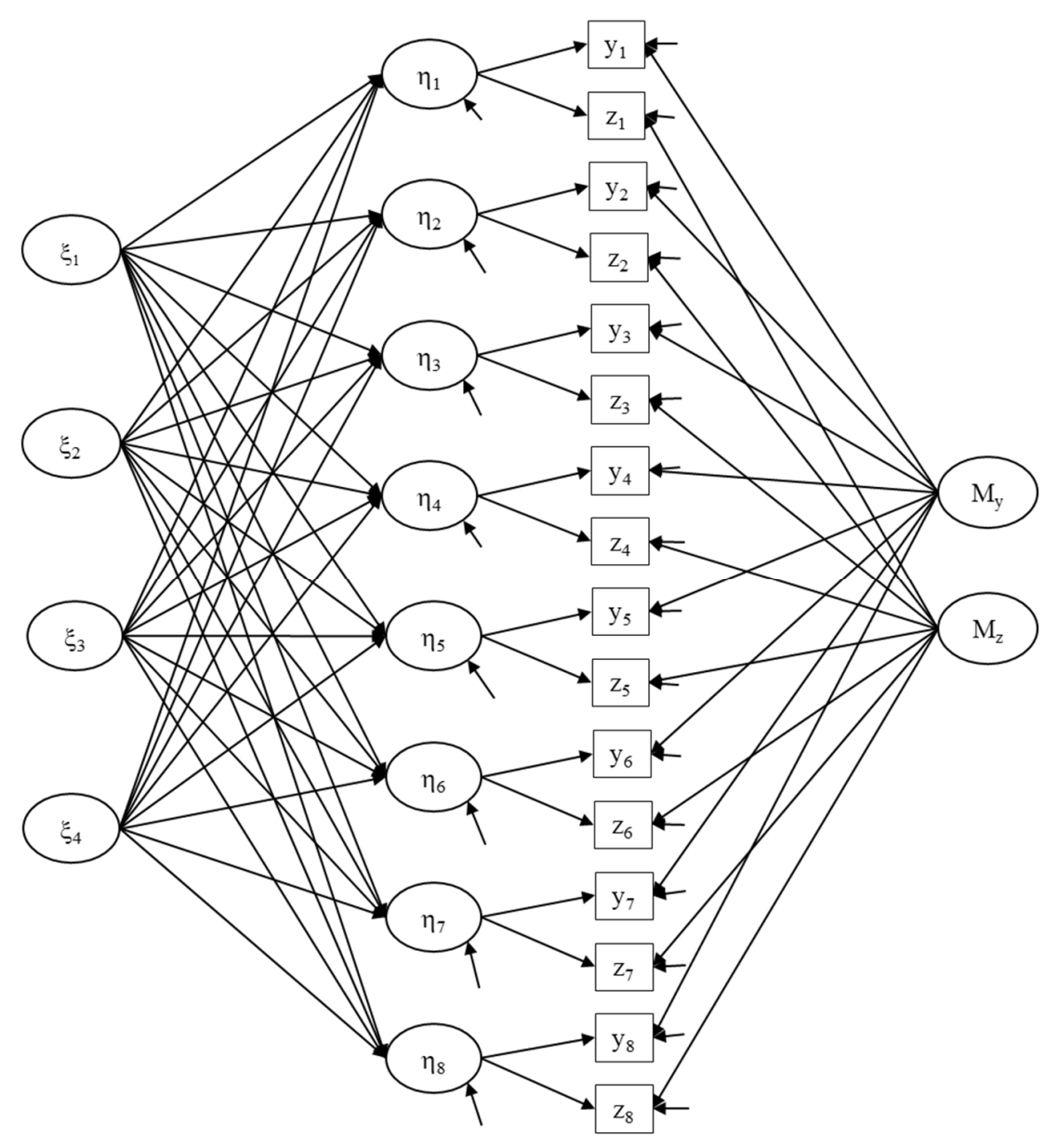

Figure 3. SEMWISE model extension with multiple indicators for the dependent variable and two method factors. Factor covariance terms are omitted for readability. 


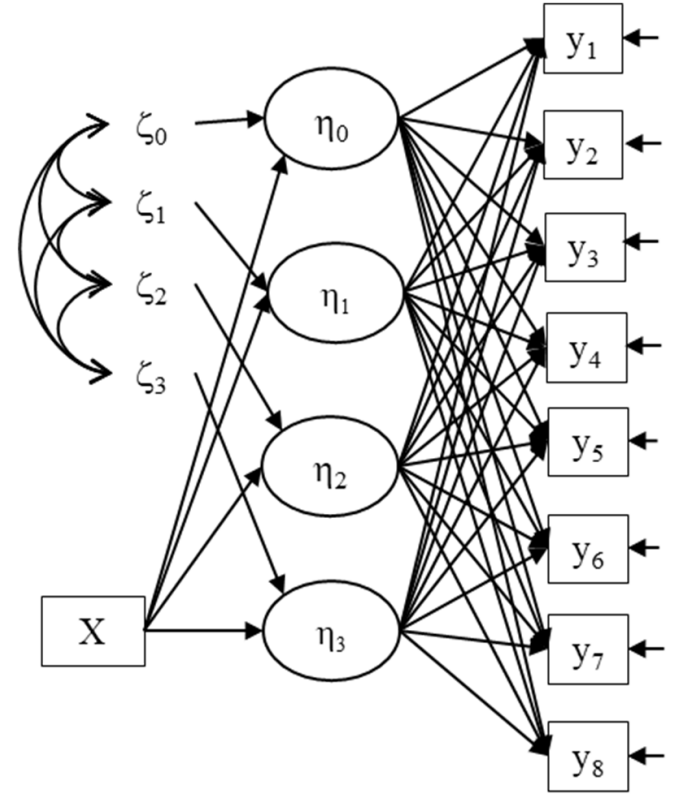

(a)

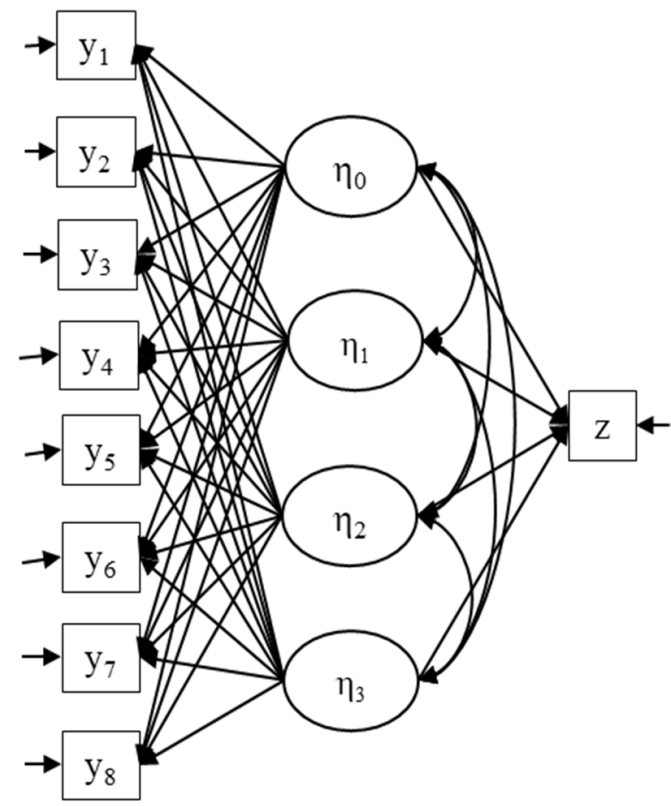

(b)

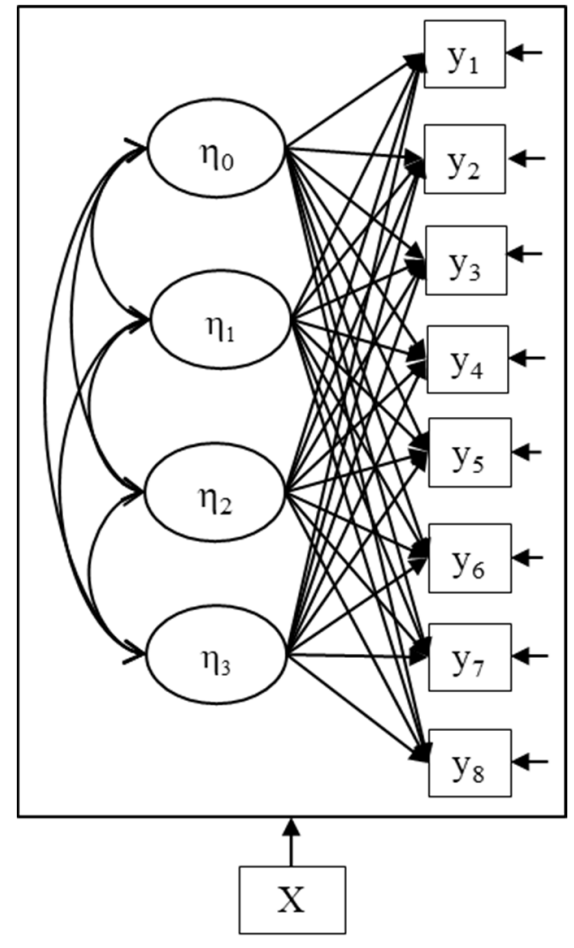

(c)

Figure 4. SEMWISE model extensions with (a) an observed antecedent variable, (b) observed outcome variable, (c) grouping variable. 


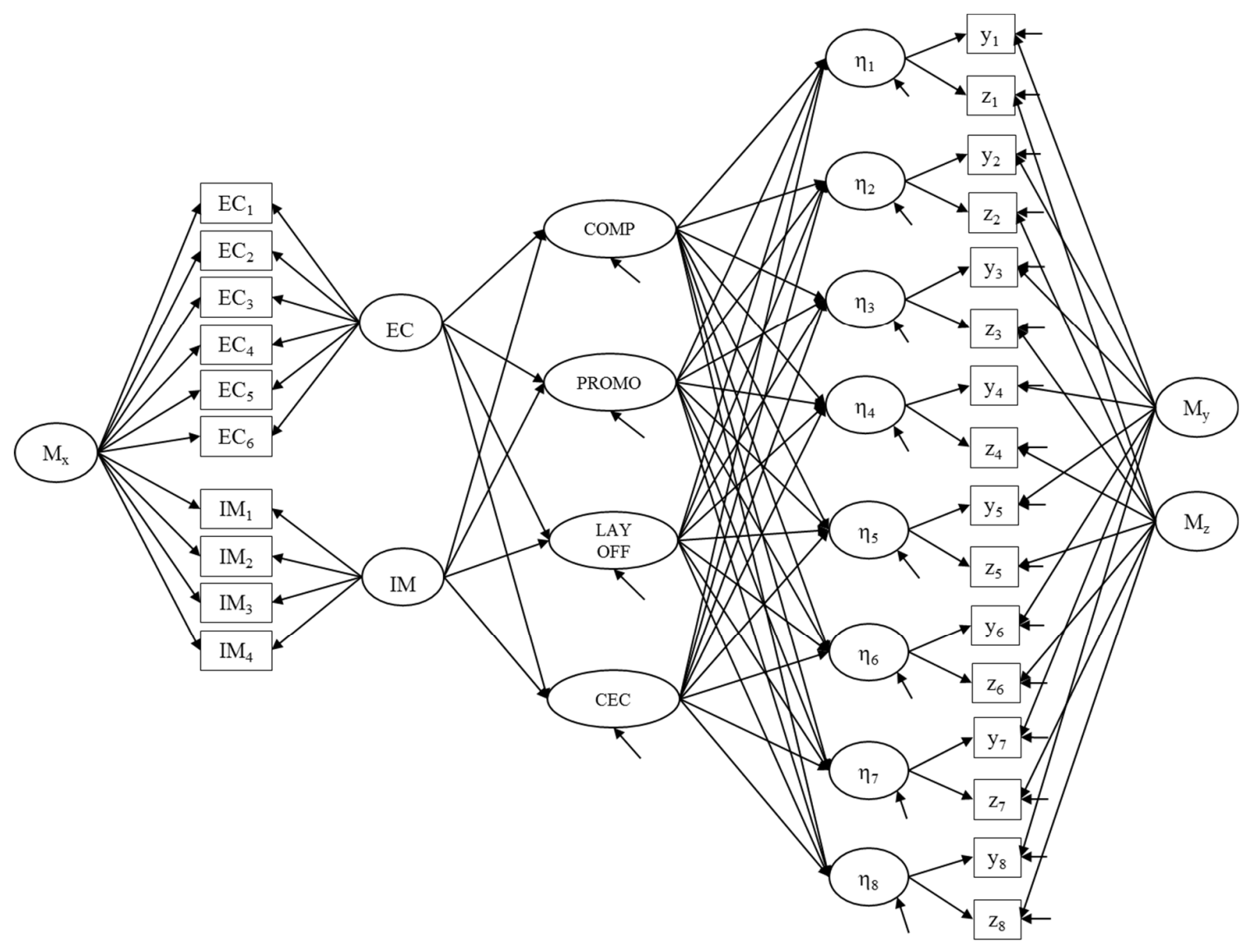

Figure 5. SEMWISE model extension with latent antecedents and multiple method factors (empirical application 2). Factor covariance terms are omitted for readability. 
1. Model specification. Specify the core model, accounting for the following:

a) Grouping:

If data are collected from different groups of respondents that need to be compared, specify the grouping variable an subsequent analyses in multi-group mode.

b) Multi-indicator measurement:

If multiple ratings for each profile have been collected, specify a second-order factor structure with method-specific factors.

c) Weight factor specification:

- Specify the intercept and weight factors.

- If some attributes have more than two levels, select an appropriate specification: If the levels are nominal, (q weight factors are needed for q levels. If the levels are (assumed to be) ordinal or metric, the researcher may use a linear coding scheme (allocated coding) for simplicity, but it is necessary to test whether this assumptic appropriate (by evaluating global and local model fit).

d) Residual variance specification: Test whether residual variance terms can be set to equality across the different profil by comparing a model without the equality constraint to a model with the equality constraint imposed and evaluating deterioration in fit (e.g., using a $\chi^{2}$ difference test).

2. Model evaluation. Evaluate the core model:

a) Evaluate global model fit (based on $\chi^{2}$ and alternative fit indices such as CFI, TLI, RMSEA, SRMR).

b) Check for local misfit (e.g., based on modification indices) to identify specific model constraints that may be problen

c) Verify that parameter estimates are meaningful (e.g., no negative variance estimates).

d) Verify that explained variance in the ratings (or rating factors in the case of multi-indicator models) is satisfactory (ty $\left.\mathrm{R}^{2}>.50\right)$.

3. Model adaptation. Add interaction effects if required:

Test models with interaction terms and evaluate model fit relative to a model without the interaction terms if one or more of following conditions hold: (a) the theoretical model requires interaction terms, (b) the explained variance in the ratings is unsatisfactory, (c) model fit in step 2 is unsatisfactory, and/or (d) indices of local misfit (esp. modification indices) suggest th for profiles that share the same combination of attribute levels are more strongly correlated than is implied by the model.

4. Model integration with other variables. Integrate the core SEMWISE model into a broader nomological network if applicable

a) Interpret the variance estimates of the weight factors. For weight factors with a non-zero variance, go to step 4b. For factors with non-significant variance, set the variance to zero; step $4 \mathrm{~b}$ does not apply for these weight factors.

b) Add antecedents, correlates and outcome variables to the model.

c) Multi-group comparison. In a multi-group setting, test for between-group invariance of the parameters of interest (es) factor means, variance and covariance terms, and regression weights linking weight factors to other variables).

5. Model interpretation.

Interpret the parameter estimates and report.

Figure 6. Suggested SEMWISE modeling steps. 


\section{Appendix A}

Comparison of the SEMWISE and MLM analysis approaches

This appendix compares the SEMWISE and multi-level modeling (MLM) approaches in terms of data setup, data analysis, and (interpretation of) results as applied to the stylized example shown in Figure 1 of the main text. Figure A1 shows how the data file is structured in the MLM approach (left-hand panel) compared to the SEMWISE approach (right-hand panel), using the four responses of the fictitious respondent in Figure 1 in the main text as an example.

\begin{tabular}{|c|c|c|c|c|}
\hline \multicolumn{5}{|c|}{ Multilevel data setup } \\
\hline Respondent & Profile & $\begin{array}{l}\text { Profile } \\
\text { warmth }\end{array}$ & $\begin{array}{c}\text { Profile } \\
\text { competence }\end{array}$ & Rating \\
\hline 1 & 1 & 1 & -1 & 2 \\
\hline 1 & 2 & -1 & 1 & 4 \\
\hline 1 & 3 & 1 & 1 & 5 \\
\hline 1 & 4 & -1 & -1 & 1 \\
\hline 2 & 1 & $\ldots$ & $\ldots$ & $\ldots$ \\
\hline 2 & 2 & $\ldots$ & $\ldots$ & $\ldots$ \\
\hline$\ldots$ & ... & $\ldots$ & $\ldots$ & $\ldots$ \\
\hline
\end{tabular}

\begin{tabular}{|c|r|r|r|r|}
\hline \multicolumn{6}{|l|}{ SEMWISE data setup } \\
\hline \hline Respondent & $\begin{array}{c}\text { Rating for } \\
\text { profile 1 }\end{array}$ & $\begin{array}{c}\text { Rating for } \\
\text { profile 2 }\end{array}$ & $\begin{array}{c}\text { Rating for } \\
\text { profile 3 }\end{array}$ & $\begin{array}{c}\text { Rating for } \\
\text { profile 4 }\end{array}$ \\
\hline 1 & 2 & 4 & 5 & 1 \\
\hline 2 & $\ldots$ & $\ldots$ & $\ldots$ & $\ldots$ \\
\hline 3 & $\ldots$ & $\ldots$ & $\ldots$ & $\ldots$ \\
\hline
\end{tabular}

Figure A1. Data setup in a simplified policy capturing study.

As shown in Figure A1, in the MLM dataset, respondents' reactions to the four profiles (obtained by crossing the two levels of warmth with the two levels of competence) are specified on four separate lines of data (i.e., each line represents the rating of one profile, with multiple lines for each respondent). In addition, the profile attribute levels are represented by two additional variables, one containing the effect-coded attribute levels for warmth for each profile, the other containing the effect-coded attribute levels for competence for each profile. The profiles' attribute levels are repeated across respondents, but the ratings are respondent-specific. For the SEMWISE approach, each participant has one line of data and there are four columns of ratings corresponding to the ratings of the four profiles. In other words, MLM requires data in long format, SEMWISE requires data in wide format. In 
the SEMWISE approach, the attribute levels of the profiles are not part of the dataset but are specified as fixed model parameters in the analytical model (see the loadings in Figure 1).

In the MLM approach, the ratings of the teammate profiles are regressed on each profile's warmth and competence level, where (for the sake of this example) effect coding is used to represent the profiles (i.e., -1 represents a low level, +1 represents a high level of an attribute). The estimated regression coefficients are the weights (or utilities) that respondents attach to warmth and competence, which are inferred from the overall ratings of the profiles. In other words, the overall rating of a profile is decomposed into the weights of the attribute levels that characterize the profile. It can be shown that when using MLM, the estimated regression coefficients, which are allowed to vary across respondents, are a compromise between (or weighted combination of) two types of regressions, a pooled regression and individual-level regressions (Gelman \& Hill, 2007). In a pooled regression, it is assumed that all respondents assign the same weights to warmth and competence when rating teammates, so the regression coefficients are fixed to be equal across respondents. In an individual-level regression, it is assumed that each respondent assigns idiosyncratic weights to warmth and competence when rating teammates, so a separate regression equation is estimated for each respondent across the rated profiles (four in the present case). If there is little variability in coefficients across respondents, the MLM estimates will be close to the pooled estimates, but as the variability in coefficients across respondents increases, the MLM estimates move toward the individual-level estimates. The advantage of the MLM regression coefficients is that, on the one hand, the limiting assumption of effect homogeneity is relaxed and that, on the other hand, the estimates tend to be more stable than the individual-level regression coefficients (which are often based on a relatively small number of distinct profiles).

In the SEMWISE approach, the estimated weights are the same as in the MLM approach, but the way in which the model is specified and the weights are estimated is quite different. Instead of regressing teammate ratings on warmth and competence, one specifies a 
factor model in which the ratings of the four experimental profiles are treated as the observed indicators of three latent factors: an intercept factor, a weight factor for warmth, and a weight factor for competence. In order to estimate a factor model, the data have to be structured differently, as shown in Figure A1.

Although the SEMWISE model is a factor model, it should be noted that the SEMWISE factor model differs from a conventional factor model in that the factor loadings are fixed to particular values (analogous to the effect-coded independent variables in the MLM approach) and each indicator (i.e., each profile rating) loads on multiple factors, the reason being that each profile rating is assumed to be a function of the conjoint effect of multiple weight factors (e.g., the rating of the profile with high warmth and high competence depends on the weight that respondents attach to warmth and the weight that respondents attach to competence).

The weight factor scores in the SEMWISE approach are identical to the regression coefficients estimated in the MLM approach. Specifically, the latent intercept factor captures the average rating that each respondent gives to the entire set of profiles. The latent intercept factor has unit loadings on the four indicators (the ratings of the four profiles) and corresponds to the intercept term in the MLM approach. The four profile ratings have loadings on the latent warmth and competence weight factors, and these loadings correspond to the profiles' effect codes (for warmth and competence) in the MLM approach. Thus, both approaches model each profile rating as a function of its effect-coded attribute levels and the respective weights (regression coefficients in MLM, weight factor scores in SEMWISE) of these attribute levels. Since the loadings are effect-coded as -1 for low and +1 for high warmth and competence, respectively, twice the estimated factor score is the difference in the weight attached to low and high warmth or competence.

Below we provide Mplus syntax to simulate data corresponding to the example shown in Figure 1 and to analyze the data in an equivalent way using either SEMWISE or MLM 
(see Figure A1). The Mplus output shows that the parameter estimates are the same across the

MLM and SEMWISE analyses, although the terminology differs across approaches.

5. Data generation

TITLE: Data generation for SEMWISE VS. MLM (Fig 1 and Fig A1); MONTECARLO:

NAMES $=\mathrm{y} 1-\mathrm{y} 4 ;$

NOBS $=250$;

SAVE = example_wide.dat;

$\mathrm{SEED}=0$;

MODEL POPULATION:

icept BY y1-y4@1;

wfwarm BY y1@1 y2@-1 y3e1 y4@-1;

!response variables: profile rating;

!sample size

!generated data filename

!results can be replicated exactly

!true model for data generation

! intercept factor

wfcomp BY y1@-1 y2@1 y3@1 y4@-1;

! weight factor for warmth

!weight factor for competence

!factor variances are .7

icept WITH Wfwarm*.1;

icept WITH wfcomp*.1;

wfwarm WITH wfComp*.1;

$\mathrm{y} 1-\mathrm{y} 4 * 2$;

!factor covariance

!factor covariance

!factor covariance

!residual variance of response

variable

[y1-y4@0] ;

[icept*3 wfwarm*.6 wfcomp*.3];

! intercepts of response variable

! intercept and weight factor means

MODEL: y1-y4 with y1-y4;

!observed sample covariances

6. Data analysis using the SEMWISE approach

TITLE: SEMWISE analysis for the example in Figures 1 and 2;

DATA: FILE = example_wide.dat; !use the data in wide format

VARIABLE: NAMES $=y 1-y 4$;

!response variables

MODEL:

icept by y1-y4@1;

wfwarm by y1e1 y2e-1 y3e1 y4e-1;

!intercept factor

wfcomp by y1@-1 y2a1 y3a1 y4a-1;

!weight factor for warmth

$[y 1-y 4 @ 0]$;

!weight factor for competence

!response intercepts are set to zero

[icept wfwarm wfcomp];

$\mathrm{y} 1-\mathrm{y} 4(\mathrm{e})$;

!freely estimated factor means

!residual variances equal for

correspondence with MLM

OUTPUT: TECH1 STANDARDIZED;

!standardized keyword gives $\mathrm{R}^{2}$

7. Data analysis using the MLM approach

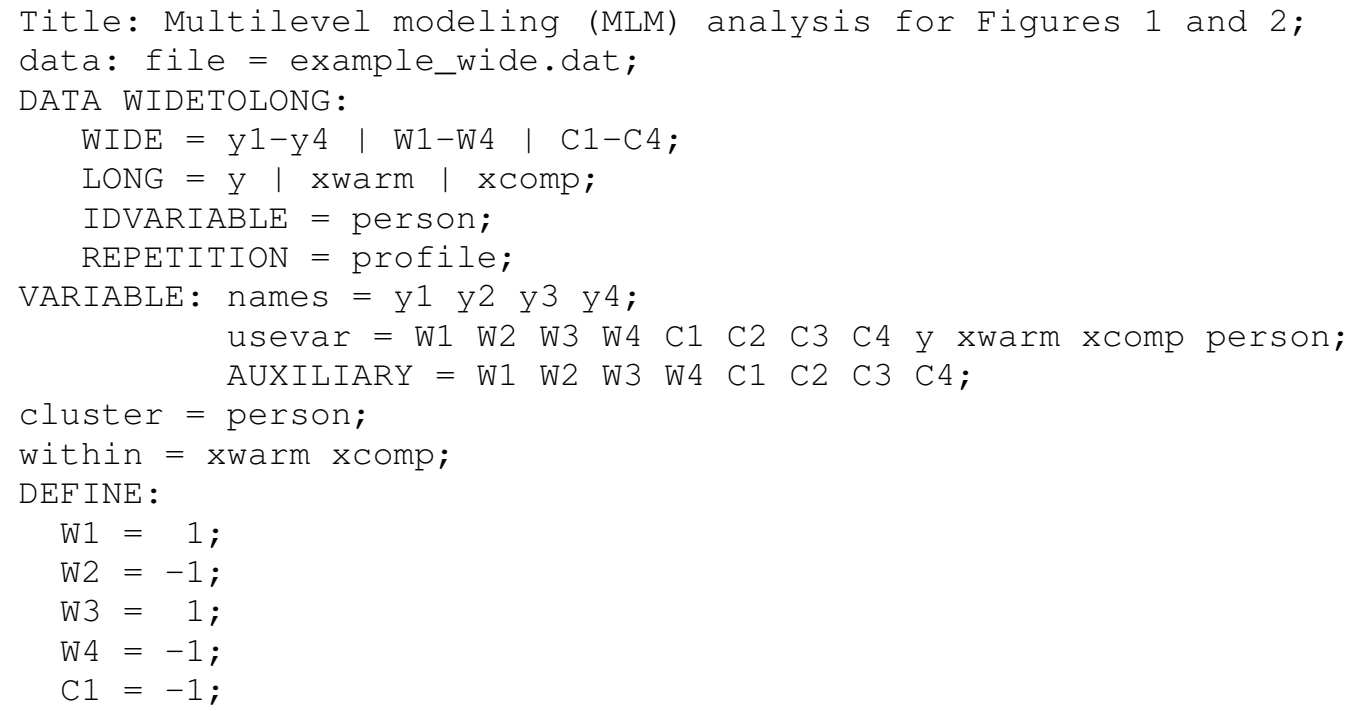




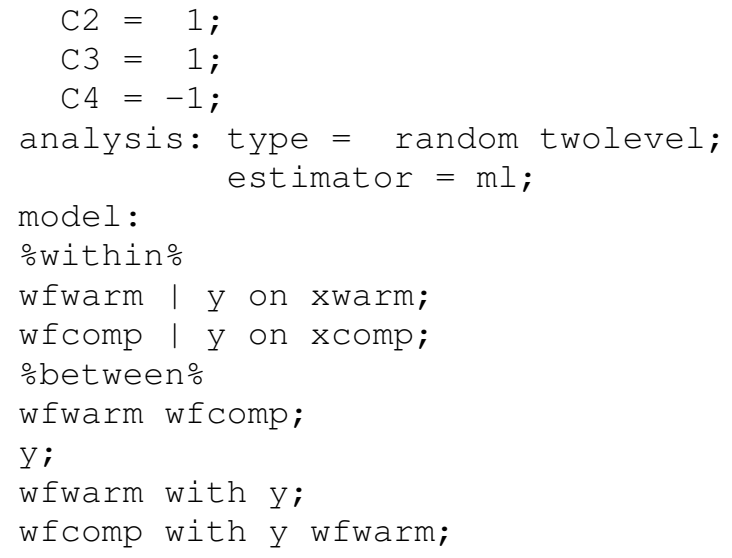

8. Selected output for both approaches

\begin{tabular}{|c|c|c|c|c|}
\hline \multicolumn{5}{|l|}{ SEMWISE } \\
\hline & Est. & S.E. & $t$ & $\mathrm{p}$ \\
\hline WEWARM & WITH & & & \\
\hline ICEPT & 0.138 & 0.049 & 2.815 & 0.005 \\
\hline WECOMP & WITH & & & \\
\hline ICEPT & 0.118 & 0.048 & 2.451 & 0.014 \\
\hline WFWARM & 0.136 & 0.049 & 2.788 & 0.005 \\
\hline Means & & & & \\
\hline ICEPT & 3.036 & 0.055 & 55.193 & 0.000 \\
\hline WFWARM & 0.545 & 0.055 & 9.855 & 0.000 \\
\hline WFCOMP & 0.294 & 0.055 & 5.364 & 0.000 \\
\hline Variano & ces & & & \\
\hline ICEP T & 0.707 & 0.068 & 10.434 & 0.000 \\
\hline WEWARM & 0.715 & 0.069 & 10.442 & 0.000 \\
\hline WFCOMP & 0.700 & 0.067 & 10.426 & 0.000 \\
\hline Residua & al Vari & & & \\
\hline Y1 & 0.196 & 0.018 & 11.180 & 0.000 \\
\hline Y2 & 0.196 & 0.018 & 11.180 & 0.000 \\
\hline Y3 & 0.196 & 0.018 & 11.180 & 0.000 \\
\hline Y4 & 0.196 & 0.018 & 11.180 & 0.000 \\
\hline
\end{tabular}

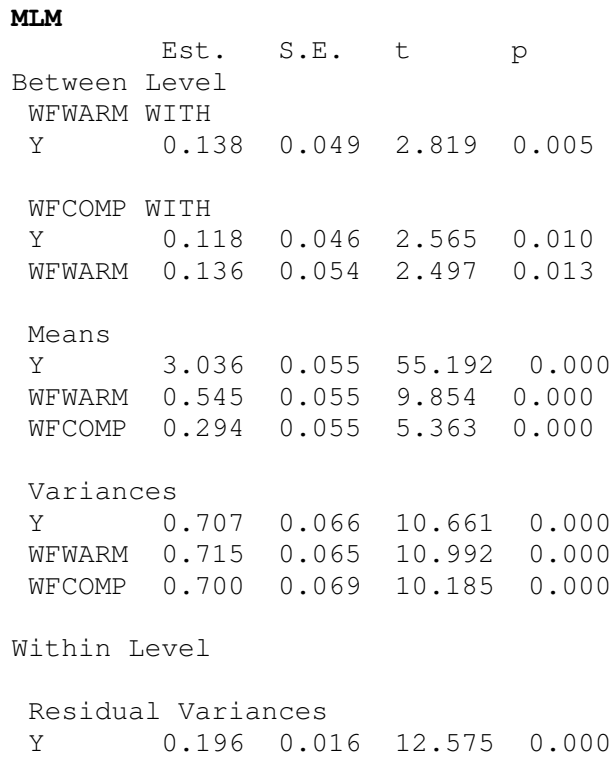




\section{Appendix B}

Correlation matrix for empirical application 1

\begin{tabular}{|c|c|c|c|c|c|c|c|c|}
\hline & 1 & 2 & 3 & 4 & 5 & 6 & 7 & 8 \\
\hline 1 Female & & & & & & & & \\
\hline $2 \mathrm{HHL}$ & .009 & & & & & & & \\
\hline 3 HLL & .039 & .481 & & & & & & \\
\hline $4 \mathrm{HHH}$ & -.023 & .420 & 297 & & & & & \\
\hline $5 \mathrm{HLH}$ & -.062 & .222 & .376 & .418 & & & & \\
\hline $6 \mathrm{LHL}$ & .055 & .547 & 382 & .358 & 103 & & & \\
\hline 7 LLL & .027 & 432 & .585 & .280 & .344 & .415 & & \\
\hline $8 \mathrm{LHH}$ & .043 & .415 & .337 & .556 & .337 & .418 & .289 & \\
\hline $9 \mathrm{LLH}$ & .045 & 169 & 398 & .362 & .595 & .188 & .459 & .414 \\
\hline
\end{tabular}

Note: Correlations based on data setup in wide format (responses to the experimental profiles correspond to variables 2 to 9). Female refers to respondent gender $(0=$ male, $1=$ female). The other variable names refer to the levels $(\mathrm{L}=\mathrm{low}, \mathrm{H}=\mathrm{high})$ of the three attributes attractiveness, warmth and competence. 


\section{Appendix C}

Correlation matrix of empirical application 2

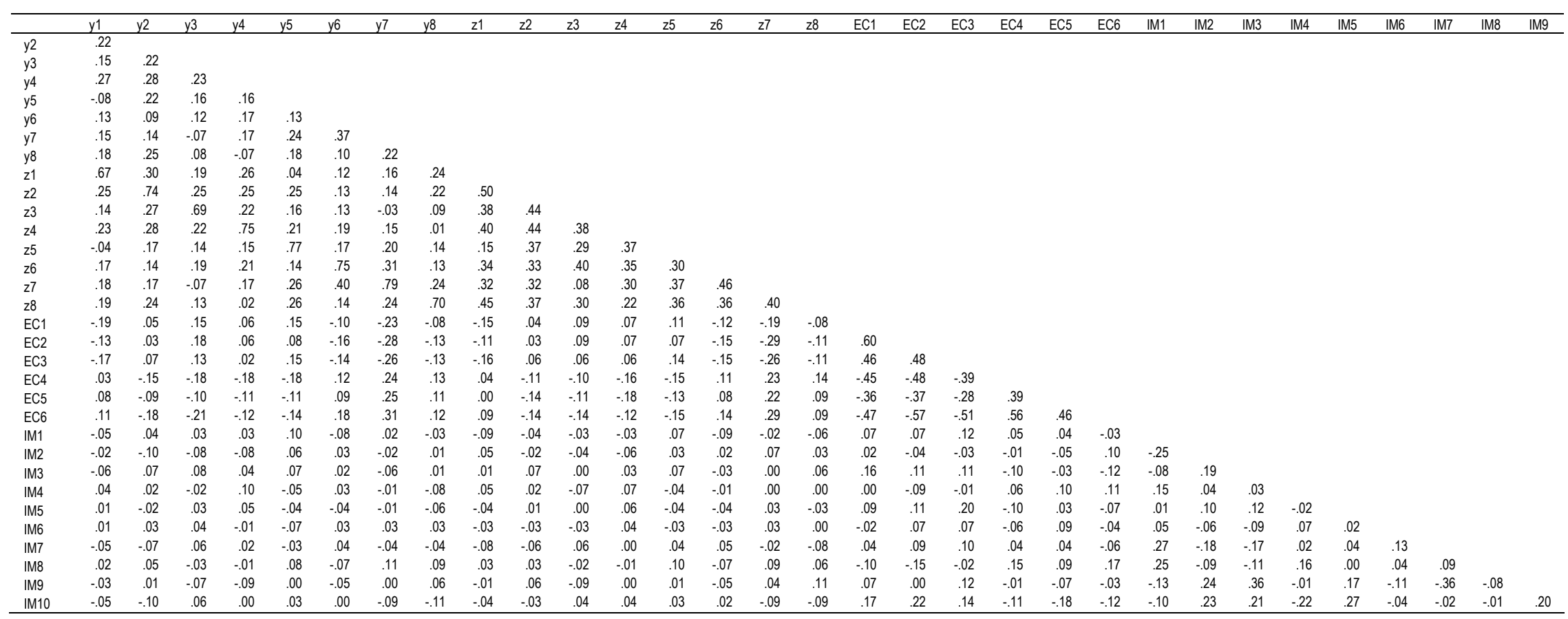

Note: Correlations based on data setup in wide format. Variables y1-y8 refer to profile ratings using the first item ('To what extent would you like to work for this company?' rated by manipulating a sliding bar linked to a smiley face with five levels ranging from 1= low to 5=high), z1-z8 to profile ratings using the second item ('How attractive do you find this company?' rated by means of a continuous sliding scale marked by five stars with scores ranging from zero to five). EC1-EC6 refer to the Environmental Concern scale items, IM1-IM10 to the Impression Management scale items. 


\section{Appendix D}

Mplus, R, and LISREL syntax for analyzing the data of the SEMWISE core model and several extensions

This web appendix provides Mplus syntax for generating artificial data and Mplus, R, and LISREL syntax for analyzing the data of the

SEMWISE core model and several extensions.

\begin{tabular}{|c|c|c|c|}
\hline Mplus data generation & Mplus data analysis & $\mathrm{R}$ data analysis & LISREL data analysis \\
\hline $\begin{array}{l}\text { !Example } 1 \text { data generation; } \\
\text { Title: SEMWISE core model data generation } \\
\text { (see Figure 2); } \\
\text { montecarlo: } \\
\text { names = y1-y8; } \\
\text { nobs = 500; } \\
\text { save = semwise_core.dat; } \\
\text { model population: } \\
\text { eta0 by y1-y8@1; } \\
\text { eta1 by y1@1 y2@1 y3@1 y4@1 y5@-1 } \\
\text { y6@-1 y7@-1 y8@-1; } \\
\text { eta2 by y1@-1 y2@-1 y3@1 y4@1 y5@-1 } \\
\text { y6@-1 y7@1 y8@1; } \\
\text { eta3 by y1@1 y2@-1 y3@1 y4@-1 y5@1 } \\
\text { y6@-1 y7@1 y8@-1; } \\
\text { eta0-eta3*.7; } \\
\text { eta0 with eta1*.1; } \\
\text { eta0 with eta2*.1; } \\
\text { eta0 with eta3*.1; } \\
\text { eta1 with eta2*.1; } \\
\text { eta1 with eta3*.1; } \\
\text { eta2 with eta3*.1; } \\
\text { y1-y8*.2; }\end{array}$ & $\begin{array}{l}\text { !Example } 1 \text { data analysis; } \\
\text { Title: SEMWISE core model data analysis (see } \\
\text { Figure 2); } \\
\text { data: file = semwise_core.dat; } \\
\text { variable: names = y1-y8; } \\
\text { model: } \\
\text { eta0 by y1-y8@1; } \\
\text { eta1 by y1@1 y2@1 y3@1 y4@1 y5@-1 } \\
\text { y6@-1 y7@-1 y8@-1; } \\
\text { eta2 by y1@-1 y2@-1 y3@1 y4@1 y5@-1 } \\
\text { y6@-1 y7@1 y8@1; } \\
\text { eta3 by y1@1 y2@-1 y3@1 y4@-1 y5@1 } \\
\text { y6@-1 y7@1 y8@-1; } \\
\text { eta0-eta3*7; } \\
\text { eta0 with eta1*.1; } \\
\text { eta0 with eta2*1; } \\
\text { eta0 with eta*.1; } \\
\text { eta1 with eta2*.1; } \\
\text { eta1 with eta*.1; } \\
\text { eta2 with eta3*.1; } \\
\text { y1-y8*.5; } \\
\text { [y1-y8@0]; [eta0-eta3]; }\end{array}$ & 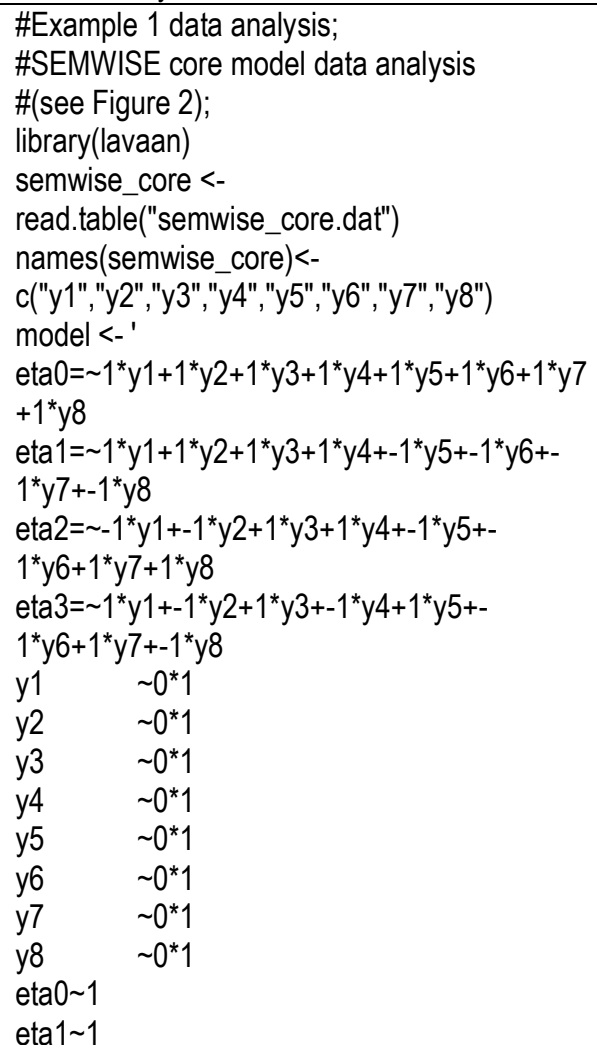 & 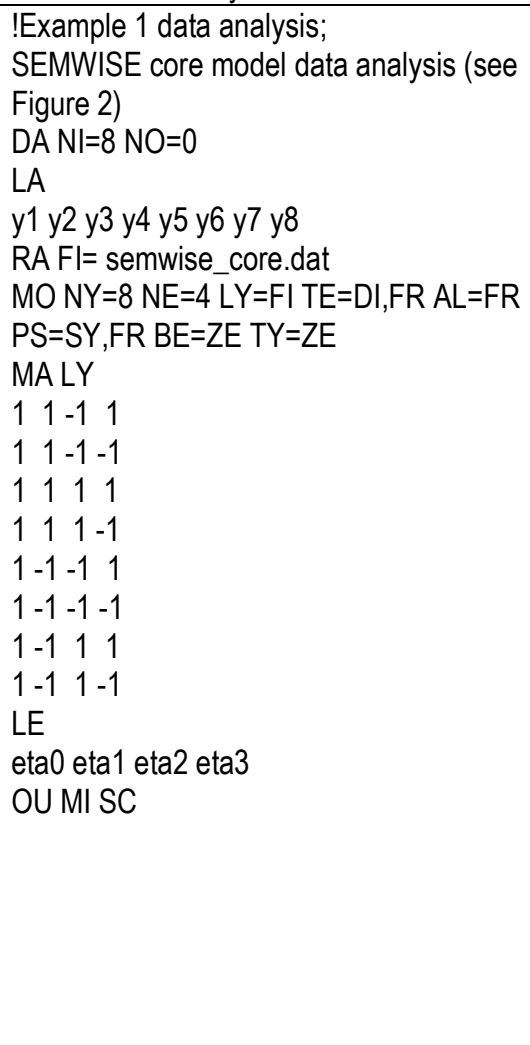 \\
\hline
\end{tabular}




\begin{tabular}{|c|c|c|c|}
\hline & & $\begin{array}{l}\text { eta2 1 } \\
\text { eta3 1 } \\
\text { ' } \\
\text { fit <- sem(model, data=semwise_core, } \\
\text { meanstructure = TRUE) } \\
\text { summary(fit) }\end{array}$ & \\
\hline !Example 3 data generation; & !Example 3 data analysis; & $\begin{array}{l}\text { \#Example } 3 \text { data analysis; } \\
\text { \#Two methods SEMWISE analysis; }\end{array}$ & !Example 3 data analysis; \\
\hline
\end{tabular}




\begin{tabular}{|c|c|c|c|}
\hline $\begin{array}{l}\text { Title: Two methods SEMWISE data } \\
\text { generation (Figure 3); } \\
\text { montecarlo: } \\
\text { names = y1-y8 z1-z8; } \\
\text { nobs = 500; } \\
\text { save = semwise2methods.dat; } \\
\text { model population: } \\
\text { My by y1-y8@1; } \\
\text { Mz by z1-z8@1; } \\
\text { eta1 by y1@1 z1@1.2; } \\
\text { eta2 by y2@1 z2@1.2; } \\
\text { eta3 by y3@1 z3@1.2; } \\
\text { eta4 by y4@1 z4@1.2; } \\
\text { eta5 by y5@1 z5@1.2; } \\
\text { eta6 by y6@1 z6@1.2; } \\
\text { eta7 by y7@1 z7@1.2; } \\
\text { eta8 by y8@1 z8@1.2; } \\
\text { ksi1 by eta1@-1 eta2@-1 eta3@-1 eta4@-1 } \\
\text { eta5@1 eta6@1 eta7@1 eta8@1; } \\
\text { ksi2 by eta1@-1 eta2@-1 eta3@1 eta4@1 } \\
\text { eta5@-1 eta6@-1 eta7@1 eta8@1; } \\
\text { ksi3 by eta1@-1 eta2@-1 eta3@1 eta4@1 } \\
\text { eta5@1 eta6@1 eta7@-1 eta8@-1; } \\
\text { ksi4 by eta1@-1 eta2@1 eta3@-1 eta4@1 } \\
\text { eta5@-1 eta6@1 eta7@-1 eta8@1; } \\
\text { ksi1-ksi4*.8; } \\
\text { ksi1 with ksi2*.1 ksi3*.1 ksi4*.1; } \\
\text { ksi2 with ksi3*.1 ksi4*.1; } \\
\text { ksi3 with ksi4*.1; } \\
\text { y1-y8*.2; } \\
\text { z1-z8*.3; } \\
\text { My*.2 Mz*.2; } \\
\text { My with Mz*.1; } \\
\text { My Mz with ksi1-ksi4@0; } \\
\text { eta1-eta8*.2; } \\
\text { [z1-z8@0]; } \\
\text { [eta1-eta8@0]; } \\
\text { [My*0 Mz*0]; } \\
\text { [ksi1*.5 ksi2*.5 ksi3*-.25 ksi4*.25]; }\end{array}$ & 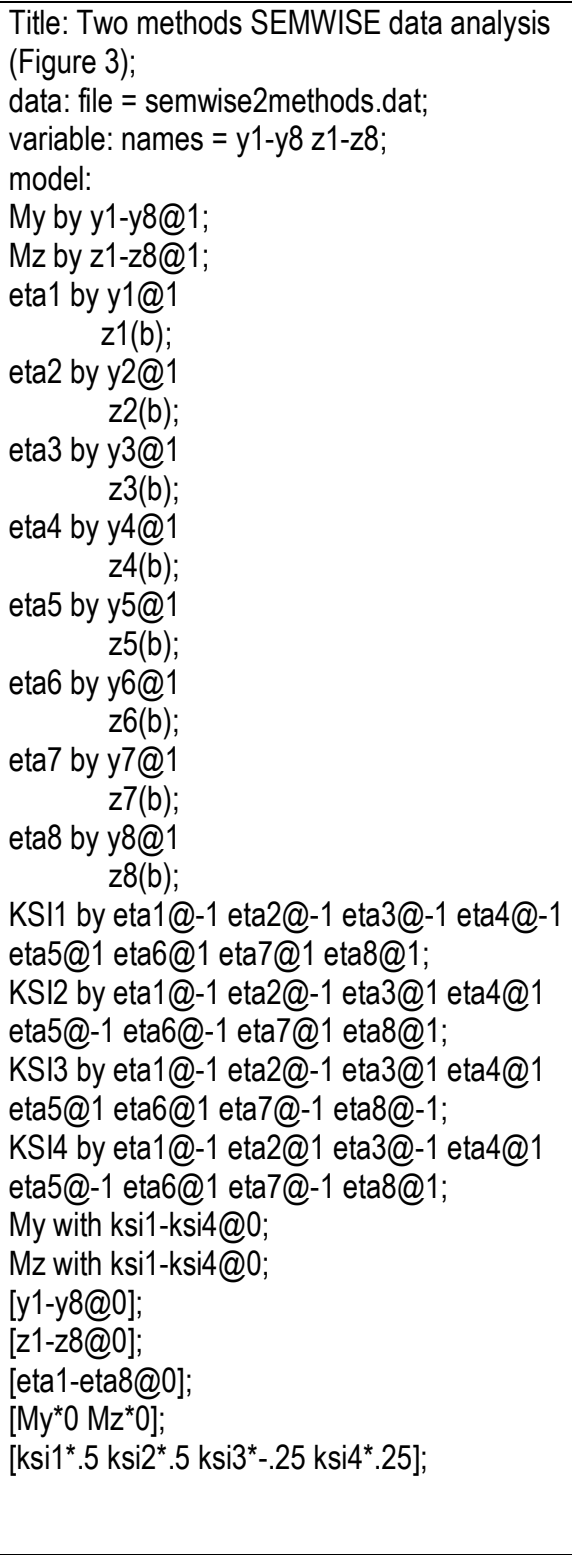 & 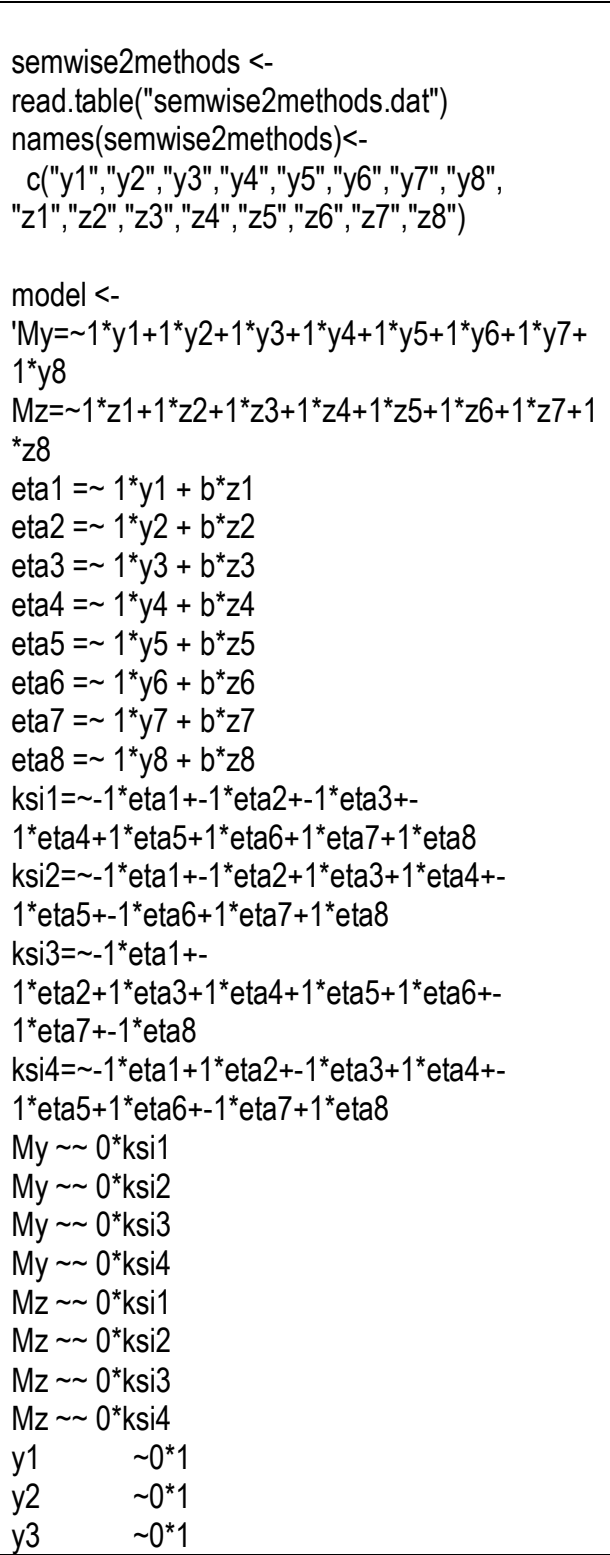 & 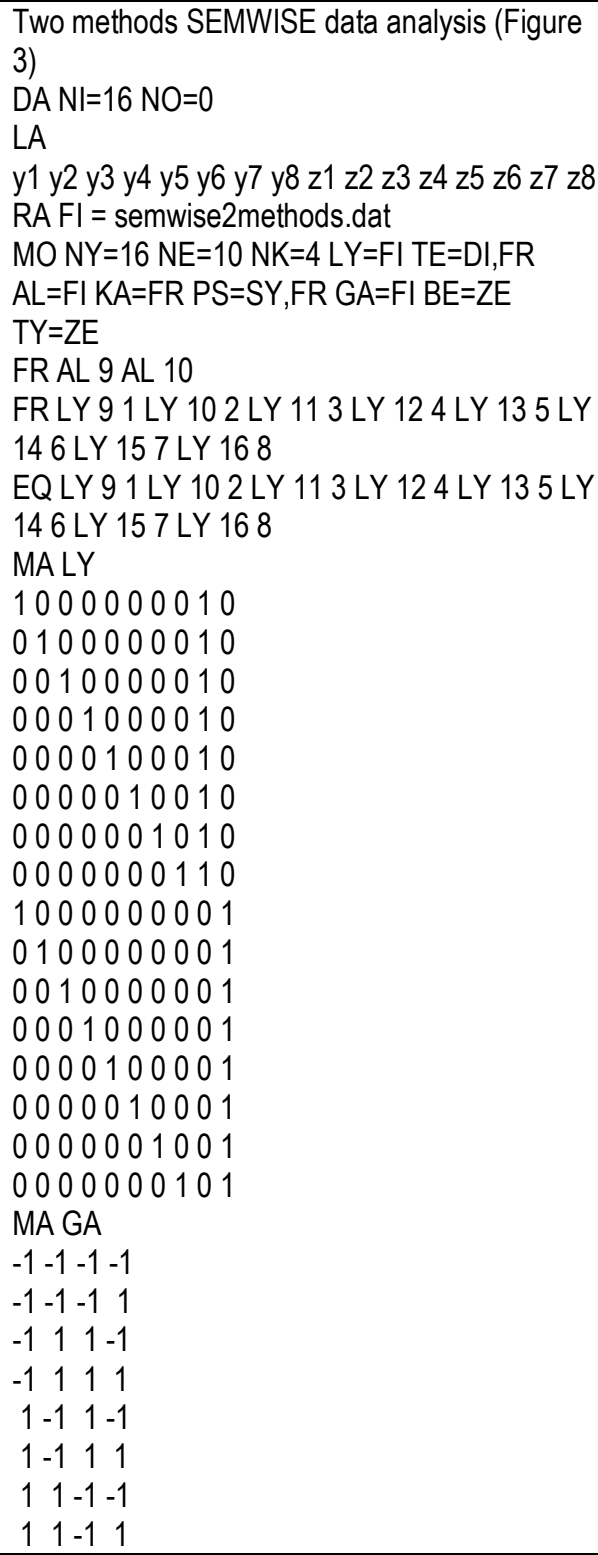 \\
\hline
\end{tabular}




\begin{tabular}{|c|c|c|c|}
\hline & & $\begin{array}{ll}\text { y4 } & \sim 0^{* 1} \\
\text { y5 } & \sim 0^{* 1} \\
\text { y6 } & \sim 0^{* 1} \\
\text { y7 } & \sim 0^{* 1} \\
\text { y8 } & \sim 0^{* 1} \\
\text { z1 } & \sim 0^{* 1} \\
\text { z2 } & \sim 0^{* 1} \\
\text { z3 } & \sim 0^{* 1} \\
\text { z4 } & \sim 0^{* 1} \\
\text { z5 } & \sim 0^{* 1} \\
\text { z6 } & \sim 0^{* 1} \\
\text { z7 } & \sim 0^{* 1} \\
\text { z8 } & \sim 0^{* 1} \\
\text { eta1 } & \sim 0^{* 1} \\
\text { eta2 } & \sim 0^{* 1} \\
\text { eta3 } & \sim 0^{* 1} \\
\text { eta4 } & \sim 0^{* 1} \\
\text { eta5 } & \sim 0^{* 1} \\
\text { eta6 } & \sim 0^{* 1} \\
\text { eta7 } & \sim 0^{* 1} \\
\text { eta8 } & \sim 0^{* 1} \\
\text { My 1 } & \\
\text { Mz 1 } & \\
\text { ksi1 1 } & \\
\text { ksi2 1 } & \\
\text { ksi3 1 } & \\
\text { ksi4 1 } & \\
\text { 1 } & \\
\text { fit <- sem(model, data=semwise2methods, } \\
\text { meanstructure = TRUE) } \\
\text { summary(fit) }\end{array}$ & 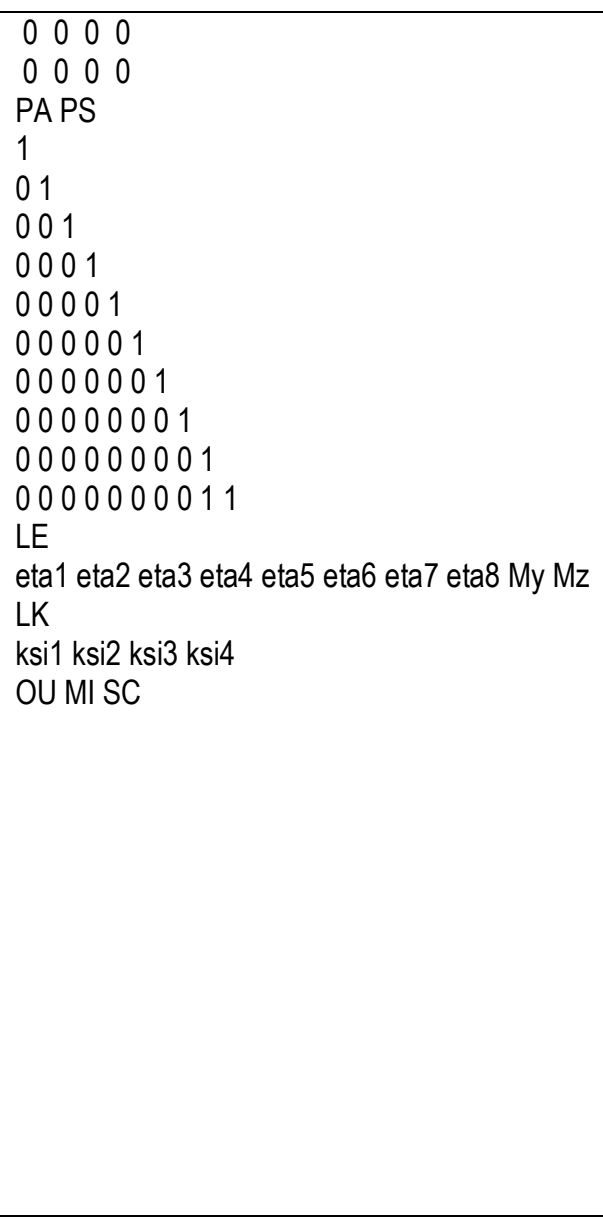 \\
\hline $\begin{array}{l}\text { !Example } 4 \text { data generation; } \\
\text { Title: Two-group SEMWISE mimic (see Figure } \\
\text { 4); } \\
\text { montecarlo: } \\
\text { names = y1-y8; } \\
\text { ngroups = 2; } \\
\text { nobs = } 250 \text { 250; } \\
\text { save = semwise2g.dat; } \\
\text { model population: }\end{array}$ & $\begin{array}{l}\text { !Example 4a data analysis; } \\
\text { Title: SEMWISE analysis using binary covariate } \\
\text { (Figure 4a); } \\
\text { data: file = semwise2g.dat; } \\
\text { variable: } \\
\text { names = y1-y8 g; } \\
\text { define: g = g-1; } \\
\text { model: } \\
\text { eta0 by y1-y8@1; }\end{array}$ & $\begin{array}{l}\text { \#Example 4a data analysis; } \\
\text { \#SEMWISE analysis using binary covariate } \\
\text { (Figure 4a) } \\
\text { semwise2g <- read.table("semwise2g.dat") } \\
\text { names(semwise2g)<- } \\
\text { c("y1","y2","y3","y4","y5","y6","y7","y8", "g") } \\
\text { semwise2g[,9]<- semwise2g[,9]-1 } \\
\text { model <- ' }\end{array}$ & $\begin{array}{l}\text { !Example 4a data analysis; } \\
\text { SEMWISE analysis using binary covariate } \\
\text { (Figure 4a) } \\
\text { DA NI=9 NO=0 } \\
\text { LA } \\
\text { y1 y2 y3 y4 y5 y6 y7 y8 g } \\
\text { RA FI= semwise2g.dat } \\
\text { MO NY=8 NX=1 NE=4 LY=FI TE=DI,FR } \\
\text { KA=FR AL=FR PS=SY,FR BE=ZE TY=ZE FI }\end{array}$ \\
\hline
\end{tabular}




\begin{tabular}{|c|c|c|c|}
\hline $\begin{array}{l}\text { eta0 by y1-y8@1; } \\
\text { eta1 by y1@1 y2@1 y3@1 y4@1 y5@-1 } \\
\text { y6@-1 y7@-1 y8@-1; } \\
\text { eta2 by y1@-1 y2@-1 y3@1 y4@1 y5@-1 } \\
\text { y6@-1 y7@1 y8@1; } \\
\text { eta3 by y1@1 y2@-1 y3@1 y4@-1 y5@1 } \\
\text { y6@-1 y7@1 y8@-1; } \\
\text { eta0-eta3*.7; } \\
\text { eta0 with eta1*.1; } \\
\text { eta0 with eta2*.1; } \\
\text { eta0 with eta3*.1; } \\
\text { eta1 with eta2*.1; } \\
\text { eta1 with eta3*.1; } \\
\text { eta2 with eta3*.1; } \\
\text { y1-y8*.2; } \\
\text { model population-g2: } \\
\text { eta0*1; } \\
\text { [eta0*.5]; } \\
\text { eta1-eta3*.25; } \\
\text { [eta1*-.25 eta2*-.5]; } \\
\text { y1-y8*.8; }\end{array}$ & $\begin{array}{l}\text { eta1 by y1@1 y2@1 y3@1 y4@1 y5@-1 y6@- } \\
\text { 1 y7@-1 y8@-1; } \\
\text { eta2 by y1@-1 y2@-1 y3@1 y4@1 y5@-1 } \\
\text { y6@-1 y7@1 y8@1; } \\
\text { eta3 by y1@1 y2@-1 y3@1 y4@-1 y5@1 y6@- } \\
\text { 1 y7@1 y8@-1; } \\
\text { eta0-eta3*.7; } \\
\text { eta0 with eta1*.1; } \\
\text { eta0 with eta2*.1; } \\
\text { eta0 with eta3*.1; } \\
\text { eta1 with eta2*.1; } \\
\text { eta1 with eta3*.1; } \\
\text { eta2 with eta3*.1; } \\
\text { y1-y8*.5; } \\
\text { eta0-eta3 on g; } \\
\text { [y1-y8@0]; } \\
\text { [eta0-eta3]; } \\
\text { !Example 4b data analysis; } \\
\text { Title: Two-group SEMWISE (Figure 4c); } \\
\text { data: file = semwise2g.dat; } \\
\text { variable: } \\
\text { names = y1-y8 g; } \\
\text { grouping = g (1 = male } 2 \text { = female); } \\
\text { model: } \\
\text { eta0 by y1-y8@1; } \\
\text { eta1 by y1@1 y2@1 y3@1 y4@1 y5@-1 y6@- } \\
\text { 1 y7@-1 y8@-1; } \\
\text { eta2 by y1@-1 y2@-1 y3@1 y4@1 y5@-1 } \\
\text { y6@-1 y7@1 y8@1; } \\
\text { eta3 by y1@1 y2@-1 y3@1 y4@-1 y5@1 y6@- } \\
\text { 1 y7@1 y8@-1; } \\
\text { eta0-eta3*.7; } \\
\text { eta0 with eta1*.1; } \\
\text { eta0 with eta2*.1; } \\
\text { eta0 with eta3*.1; } \\
\text { eta1 with eta2*.1; } \\
\text { eta1 with eta3*.1; } \\
\text { eta2 with eta3*.1; } \\
\text { y1-y8*.5; }\end{array}$ & 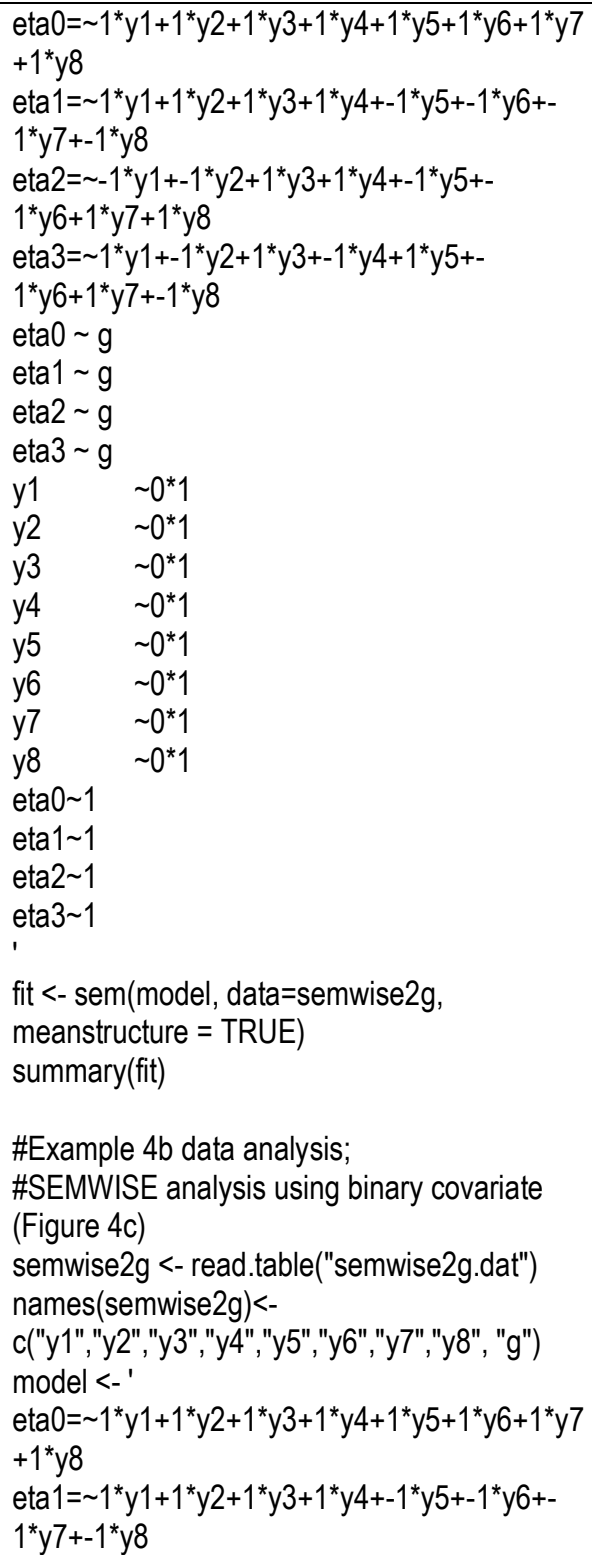 & 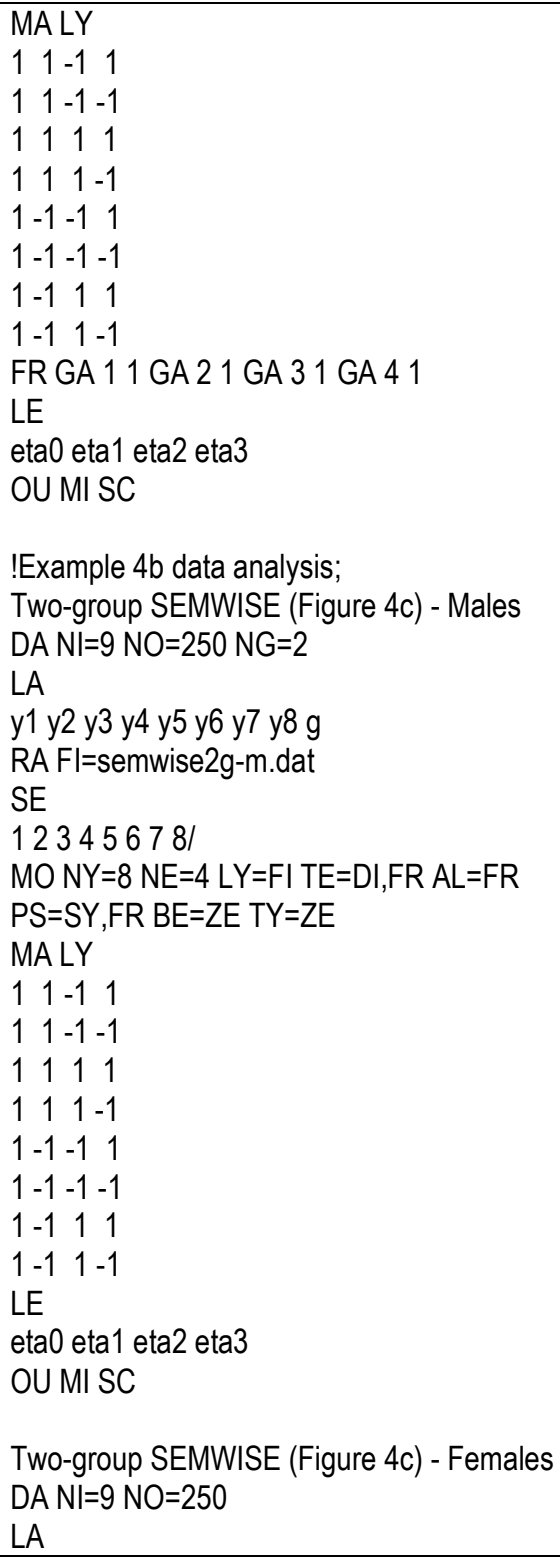 \\
\hline
\end{tabular}




\begin{tabular}{|c|c|c|c|}
\hline & $\begin{array}{l}\text { [y1-y8@0]; } \\
\text { model male:[eta0-eta3]; } \\
\text { model female:[eta0-eta3]; }\end{array}$ & 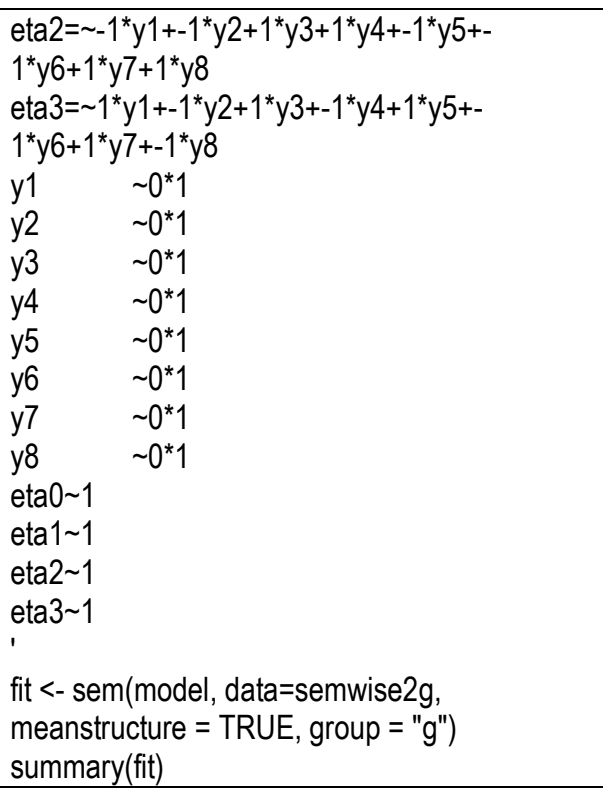 & 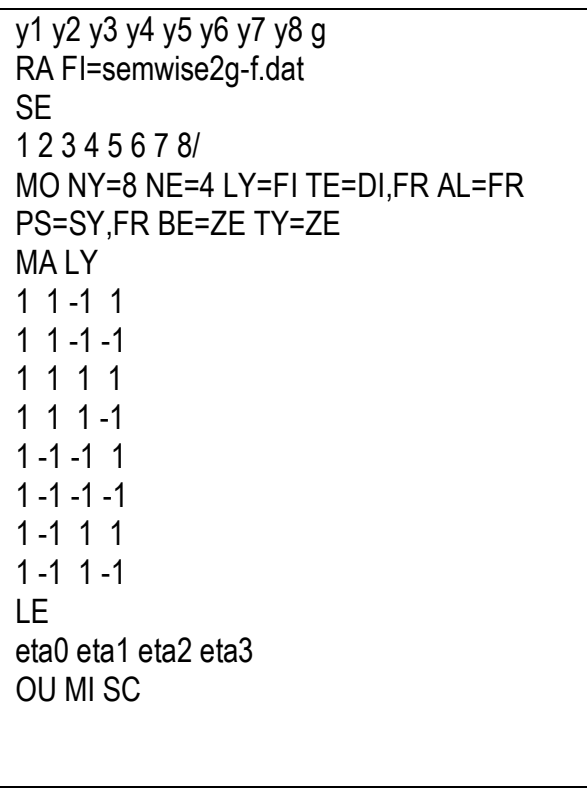 \\
\hline $\begin{array}{l}\text { Example } 5 \text { data generation; } \\
\text { Title: SEMWISE with outcome data } \\
\text { generation (Figure 4a and 4b); } \\
\text { montecarlo: } \\
\text { names = outcome y1-y4 x; } \\
\text { nobs = 500; } \\
\text { nreps = 1; } \\
\text { save = semwise_mediation.dat; } \\
\text { model population: } \\
\text { model population: } \\
\text { eta0 by y1-y4@1; } \\
\text { eta1 by y1@1 y2@-1 y3@1 y4@-1; } \\
\text { eta2 by y1@1 y2@1 y3@-1 y4@-1; } \\
\text { y1-y4*.2; } \\
\text { eta0*1 eta1* } 96 \text { eta2*.84; } \\
\text { eta0-eta2 with eta0-eta2*0; } \\
\text { outcome on eta0*0 eta1*.6 eta2*-.4; } \\
\text { outcome* }{ }^{*} .5 ; \\
\text { eta0 on } x^{*} 0 ; \\
\text { eta1 on } x^{*} .2 ;\end{array}$ & $\begin{array}{l}\text { !Example } 5 \text { data analysis; } \\
\text { Title: SEMWISE with outcome data analysis } \\
\text { (Figure 4a and 4b); } \\
\text { data: file = semwise_mediation.dat; } \\
\text { variable: names = outcome y1-y4 x; } \\
\text { model: } \\
\text { eta0 by y1-y4@1; } \\
\text { eta1 by y1@1 y2@-1 y3@1 y4@-1; } \\
\text { eta2 by y1@1 y2@1 y3@-1 y4@-1; } \\
\text { outcome on eta0-eta2; } \\
\text { [y1-y4@0]; [eta0-eta2]; [x]; } \\
\text { eta0-eta2 with eta0-eta0; } \\
\text { eta0-eta2 on } x ;\end{array}$ & 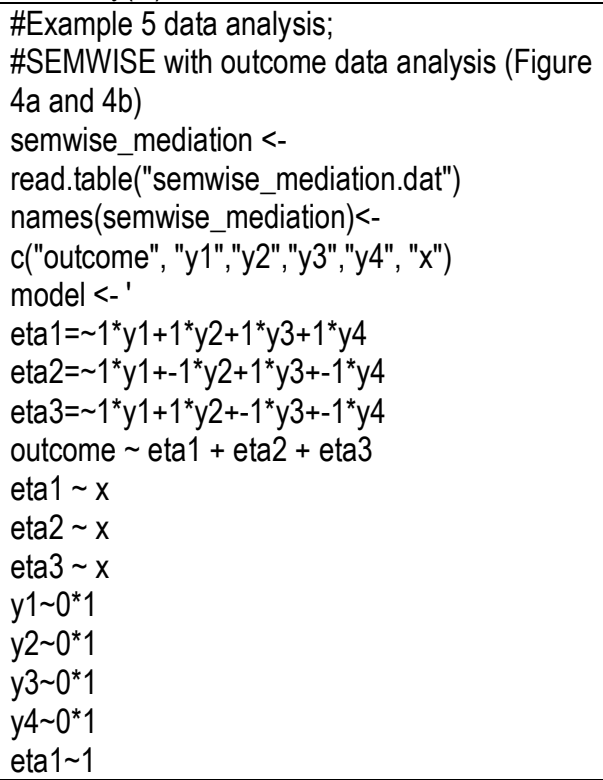 & 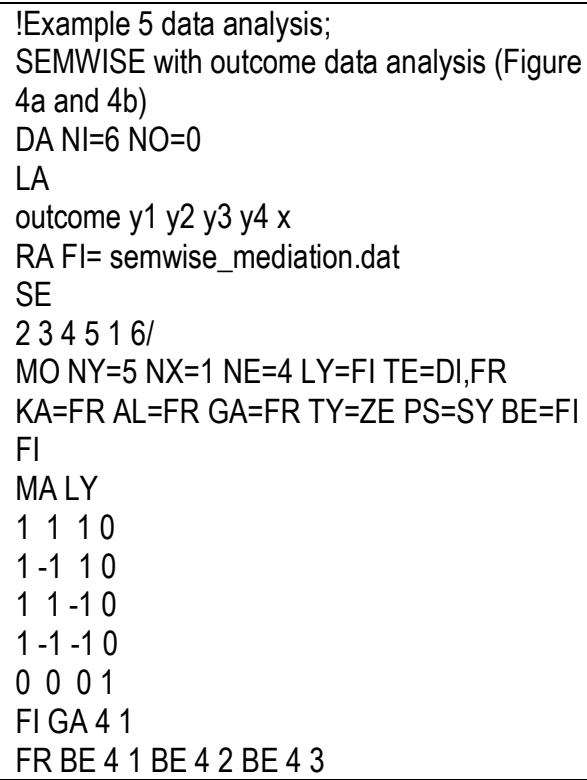 \\
\hline
\end{tabular}




\begin{tabular}{|c|c|c|}
\hline $\begin{array}{l}\text { eta2 on } x^{*} .4 \\
{\left[x^{*} 0\right] ; x^{*} 1}\end{array}$ & $\begin{array}{l}\text { eta2 1 } \\
\text { eta3 1 } \\
\text { eta2 } \sim \text { eta1 } \\
\text { eta3 eta1 } \\
\text { eta3 eta2 } \\
\text { ' } \\
\text { fit <- sem(model, data=semwise_mediation, } \\
\text { meanstructure = TRUE) } \\
\text { summary(fit) }\end{array}$ & $\begin{array}{l}\text { PA PS } \\
1 \\
11 \\
111 \\
0001 \\
\text { FI TE } 55 \\
\text { LE } \\
\text { eta0 eta1 eta2 outcome } \\
\text { OU MI SC }\end{array}$ \\
\hline
\end{tabular}

